\title{
Study of the Nanomechanics of CNTs under Tension by Molecular Dynamics Simulation Using Different Potentials
}

\author{
S. K. Deb Nath ${ }^{1,2}$ and Sung-Gaun Kim ${ }^{1}$ \\ ${ }^{1}$ Division of Mechanical and Automotive Engineering, Kongju National University, Republic of Korea \\ ${ }^{2}$ Department of Mechanical Science and Bioengineering, Graduate School of Engineering Science, Osaka University, \\ Toyonaka, Osaka 560-8531, Japan
}

Correspondence should be addressed to S. K. Deb Nath; sankar_20005@yahoo.com

Received 17 September 2013; Accepted 23 October 2013; Published 13 March 2014

Academic Editors: E. Liarokapis and M. Razeghi

Copyright (C) 2014 S. K. Deb Nath and S.-G. Kim. This is an open access article distributed under the Creative Commons Attribution License, which permits unrestricted use, distribution, and reproduction in any medium, provided the original work is properly cited.

\begin{abstract}
At four different strain rates, the tensile stress strain relationship of single-walled 12-12 CNT with aspect ratio 9.1 obtained by Rebo potential (Brenner, 1990), Airebo potential (Stuart et al., 2000), and Tersoff potential (Tersoff, 1988) is compared with that of Belytschko et al. (2002) to validate the present model. Five different empirical potentials such as Rebo potential (Brenner, 1990), Rebo potential (Brenner et al., 2002), Inclusion $L J$ with Rebo potential (Brenner, 1990), Airebo potential (Stuart et al., 2000), and Tersoff potential (Tersoff, 1988) are used to simulate CNT subjected to axial tension differing its geometry at high strain rate. In Rebo potential (Mashreghi and Moshksar, 2010) only bond-order term is used and in Rebo potential (Brenner et al., 2002) torsional term is included with the bond-order term. At high strain rate the obtained stress strain relationships of CNTs subjected to axial tension differing its geometries using five different potentials are compared with the published results and from the comparison of the results, the drawback of the published results and limitations of different potentials are evaluated and the appropriate potential is selected which is the best among all other potentials to study the elastic, elastic-plastic properties of different types of CNTs. The present study will help a new direction to get reliable elastic, elastic-plastic properties of CNTs at different strain rates. Effects of long range Van der Waals interaction and torsion affect the elastic, elastic-plastic properties of CNTs and why these two effects are really needed to consider in bond-order Rebo potential (Brenner, 1990) to get reliable elastic, elastic-plastic properties of CNTs is also discussed. Effects of length-to-diameter ratio, layering of CNTs, and different empirical potentials on the elastic, elastic-plastic properties of CNTs are discussed in graphical and tabular forms with published results as a comparative manner to understand the nanomechanics of CNTs under tension using molecular dynamics simulation.
\end{abstract}

\section{Introduction}

A variety of new intriguing materials have been discovered and synthesized in the last two decades which have caused phenomenal change in the area of materials science and among them one is the class of carbon compounds referred to fullerene nanotubes. Carbon nanotubes can be made of as rolling up sheets of graphite that are sometimes crapped on each end, with structures that vary depending on the conditions under which they are synthesized. They are singlewalled $[1,2]$ with diameters as small as about $1 \mathrm{~nm}$ or multiwalled $[3,4]$ with outer diameters ranging from 5 to $350 \mathrm{~nm}$.
Since their discovery by Lijima and Lchihashi [1] carbon nanotubes (CNTs) have been the subject of extensive research due to their unique structural and electronic properties that lead to a wide range of potential applications from electronics [5] to mechanical reinforcements in composites [6]. Early studies on the mechanical properties of CNT were carried out considering compression test [7, 8]. Yakobson et al. [7] first investigated the instability of single wall CNT under axial compression, bending, and torsional deformation using continuum shell model. Yakobson et al. [9] studied the behavior of CNTs under a high strain rate using Tersoff-Brenner's reactive empirical bond-order (REBO) potential $[10,11]$. 
Sinnott et al. [12] estimated the theoretical Young's moduli for carbon fibers composed of single-walled fullerene nanotubes aligned in the direction of the tubule axis. They also investigated a new carbon composite composed of layered nanotubule fibers and diamond and they observed that this composite had a high-modulus, low-density material that was quite stable to shear and other distortions. Liew et al. [13] examined the elastic and elastic-plastic properties of carbon nanotubes under axial tension using second generation of reactive empirical bond-order potential coupled with the Lennard-Jones potential at high strain rate. In their analysis, plastic regions are not clear because in most of the analysis of CNT it was observed that before breaking the stiffness is the highest which contradicts the reliability of their solution due to the mismanagement of the application of the Van der Waals interactions. They did not minimize CNT structure to reach its minimum energy before applying tensile loading at its both ends. Besides, Liew et al. [13] did not include temperature effect in the theoretical Rebo potential [11] and Van der Waals interaction for analyzing the elastic, elastic-plastic properties of CNTs by molecular dynamic simulation. So, the elastic-plastic properties obtained by Liew et al. [13] by molecular dynamics simulation are not beyond doubtful quality of solutions. Molecular dynamics simulations of tensile pulling of carbon nanotubes (SWCNTs) (both armchair and Zigzag configurations) were conducted using the Brenner potential to investigate the variation of six-carbon bond lengths and bond angles of the hexagons in single-walled carbon nanotubes (SWCNT) as a function of tensile strain [14]. For armchair CNTs, the brittle fracture is observed and the ultimate strength of CNTs is many times to that of CNTS obtained by Liew et al. [13]. The stress strain relationship of armchair CNT using Rebo potential by the tension test in molecular dynamics environment by Agrawal et al. [14] contradicts the elastic-plastic region of CNT by Liew et al. [13] although in his analysis Van der Waals interactions are absent. Wang and Vijayaraghavan [15] studied the buckling characteristics of several curved forms of single-walled carbon nanotubes by molecular dynamics simulations using Rebo potential [11] and Van der Waals interaction. Tserpes and Papanikos [16] proposed a threedimensional finite element model for armchair, Zigzag, and chiral single-walled carbon nanotubes which is based on the assumption that carbon nanotubes, when subjected to loading, behave like space-frame structures. The bonds between carbon atoms are considered as connecting load carrying members while the carbon atoms as joints of the members [16]. To create the FE models, nodes are placed at the locations of carbon atoms and the bonds between them are modeled using three-dimensional elastic beam elements and the elastic properties of armchair, Zigzag, and chiral single-walled carbon nanotubes are obtained which are compared with those of experimental results to verify the FE model [16]. Salvetat-Delmotte and Rubio [17] concluded that CNTs have indeed a great potential as reinforcing elements for composites. Coto et al. [18] studied the effect of carboxylation on axial Young's modulus of carbon nanotubes using molecular dynamics simulation. COMPASS force field is used to model the interatomic interactions in single wall
(SWCNT) and multiwall carbon (MWCNT) with different amount of $-\mathrm{COOH}$ group attached to their surfaces [18]. Mashreghi and Moshksar [19] determined the structural parameters of several armchair single-walled carbon nanotubes such as potential energy as a function of bond length and bond angles, respectively, using molecular dynamics simulations. Tang et al. [20] synthesized carbon nanotube polymer composites mixing carbon nanotubes and high density polyethylene by using the melt processing method and they observed that the stiffness, peak load, and work to failure for the composite films increase with the increase of MWCNT content. Sammalkorpi et al. [21] studied how the Young's modulus and tensile strength of nanotubes depends on defects employing molecular dynamics simulation and continuum theory. The Young's modulus of nanotubes with defects will essentially be the same unless the number of vacancy concentration is extremely high [21]. On the other hand, the tensile strength will substantially drop due to the quasi-one-dimensional atomic structure of SWNTs already if a single vacancy is present; the tensile strength of a SWNT is governed by the weakest segment of the tube [21]. Wang et al. [22] observed that composites showed higher mechanical properties such as the Young's modulus and tensile strength of the SWNT/epoxy composite rise with the increase of SWNT concentration. The Young's modulus and yield strength have been doubled and quadrupled for composites with, respectively, 1 and $4 \mathrm{wt} \%$ of nanotubes compared to the pure resin matrix samples [23]. A recent research illustrates qualitative relationship between Young's modulus of a CNT and the amount of disorder in the atomic structure of the walls [24]. Lin and Chen [25] evaluated the effective mechanical properties of CNT-based composites using a 3D nanoscale representative volume element based continuum mechanics and the finite element method. Hernández-Pérez and Avilés [26] investigated the influence of the interphase on the effective properties of carbon nanotube composites using finite element analysis and elasticity solutions for representative volume elements. Micro Pt wire exhibits higher strength as compared to its bulk due to decreasing size which is observed from the experimental study of bending test of Pt microwire with the help of finite element analysis [27]. In the very short range of diameters in Au nanowires, Young's modulus and yield strength increase with the decreasing of their diameters which are clearly observed in the bending and tension test of Au nanowires by molecular dynamics simulations [28]. Due to having small sizes of diameters in CNTs and as a result of increasing their surface energy, their strength and stiffness are many times to those of their conventional bulk materials which are made of $\mathrm{C}$ elements.

Due to having high stiffness and light weight, CNT is a promising candidate in nanocomposites to increase their strength and stiffness. Nowadays people are concentrating to evaluate the actual elastic, elastic-plastic properties of CNTs experimentally and theoretically. But handling CNT in experimental way is a difficult task and the obtained mechanical properties by the experimental way are beyond actual quantitative measures. To obtain the actual quantitative mechanical properties, theoretical approach is only an alternative approach. Researchers are trying to study CNT by 
quantum ab initio calculations at a very small scale and on the other hand classical molecular dynamics based on empirical potentials are being used to study large size CNT. Besides, very recently, people are trying to study CNTs using finite element and finite volume approach considering continuum approach. Classical molecular dynamics are the only reliable method to get reliable mechanical properties of CNTs, but it depends on the potentials. There are different potentials $[10,11,29,31]$ which are being widely used to obtain elastic, elastic-plastic properties of CNT. To predict actual elasticplastic and fracture region, selections of good potentials are necessary. In most of the studies, temperature effect is not included which is beyond practical condition. Firstly the elastic, elastic-plastic response of single-walled 12-12 CNTs with aspect ratio 9.1 subjected to a uniform axial loading at its both ends at four different strain rates obtained by Rebo potential [11], Airebo potential [29], and Tersoff potential [10] is compared with that of Belytschko et al. [30] to validate the present model. In the present study, we study the mechanical behaviors of CNTs subjected to a uniform tensile load at its opposite ends at high strain rate using five different potentials by molecular dynamics simulation as a comparative manner with the existing published results and the limitations of the published results as well as our results obtained by different potentials are highlighted and from our analysis we try to select appropriate potential which can give more accurate elastic, elastic-plastic response of CNTs at different strain rates. Besides, effects of usual parameters length-to-diameter ratio, layering of CNTs on the elastic, elastic-plastic properties of CNTs are shown by figures and tables. We try to establish the superiority of the present technique and results of CNTs.

\section{Simulation Procedure}

At four different strain rates such as very low strain rate (stretching velocity at both ends of CNTs is $0.09 \mathrm{~m} / \mathrm{s}$ ), low strain rate (stretching velocity at both ends of CNTs is $0.5 \mathrm{~m} / \mathrm{s}$ ), moderate strain rate (stretching velocity at both ends of CNTs is $5 \mathrm{~m} / \mathrm{s}$ ), and high strain rate (stretching velocity at both ends of CNTs is $20 \mathrm{~m} / \mathrm{s}$ ), the stress strain relationship of single-walled 12-12 CNT with aspect ratio 9.1 subjected to uniform tensile load obtained by Rebo potential [11], Airebo potential [29], and Tersoff potential [10] is compared with that of Belytschko et al. [30] to validate the model. To study the elastic, elastic-plastic properties of CNT, we need its stress strain relationship up to its fracture. In this study, we also simulate single-walled 10-10, 12-12 CNTs; doublewalled (5,5), 10-10 CNTs; three-walled (5,5), 10-10 and 1515 CNTs; four-walled (5,5), 10-10, 15-15, and 20-20 CNTs using molecular dynamics simulation considering five different potentials such as Tersoff [10], Rebo potential [11], Rebo potential [31], Inclusion LJ with Rebo potential [11], and Airebo potential [29] at high strain rate. Firstly the CNT structure is minimized to get its minimum energy using different potentials before applying velocity to two groups of atoms at both ends of CNTs for all strain rates. At high strain rate tension test is carried out applying a constant velocity $20 \mathrm{~m} / \mathrm{s}$ at some atoms of both ends of the CNT in opposite directions by molecular dynamics simulation. Before applying velocity, the total force on some grouped atoms at both ends of the CNT is kept null so that extra force is not counted during stress strain relationship. Force is counted after applying velocity to a group of atoms at both end of the CNT. And the number of atoms in each group is kept equal. In the microcanonical ensemble molecular dynamics simulations of CNTs, the strain is computed by $\varepsilon=\left(L-L_{0}\right) / L_{0}$ in which $L_{0}$ and $L$ are the initial and current length of CNTs, respectively, and the stress is obtained by $\sigma=F / S$. The axial force $F$ is obtained by summing the interatomic force for the atoms at the end of CNTs and the cross-sectional area is $S=\pi d h$, where $d$ is the diameter of the CNT and $h$ is the thickness of the CNT that is taken as $h=0.335 \mathrm{~nm}$ for a 10-10 single-walled CNT, $h=0.67 \mathrm{~nm}$ for a two-walled $(5,5)$ and $10-10 \mathrm{CNT}, h=1.005 \mathrm{~nm}$ for a three-walled (5,5), 10-10, and 15-15 CNT, and $h=1.34 \mathrm{~nm}$ for a four-walled $(5,5), 10-10,15-15$, and 20-20 CNT. $L_{0} / d$ ratio of CNTs is considered as 9.1 and 4.5, respectively, in the present study. Used time step is $0.001 \mathrm{ps}$ and the whole simulation is considered at a temperature $300 \mathrm{~K}$. Using NVT canonical ensemble, temperature is controlled. Details of the used empirical potentials in molecular dynamics simulation of CNTs are discussed in the following sections for clear understanding of the readers. Due to absence of long range Van der Waals interactions and torsional effects in Tersoff potential [10] and Rebo potential [11], Rebo potential [31], and Inclusion LJ in Rebo potential [10], there is possibility of having some inaccuracies of the elastic-plastic properties of CNT when the simulation is carried out considering Tersoff potential [10], Rebo potential [11], Rebo potential [31], and Inclusion LJ with Rebo potential [11].

\section{Theoretical Formulations}

3.1. Tersoff Potential [10]. The energy E, as a function of the atomic coordinates by Tersoff [10], is taken to be

$$
E=\sum_{i} E_{i}=\frac{1}{2} \sum_{i \neq j} V_{i j} .
$$

Here $E$ is the total energy of the system, which is decomposed for convenience into a site energy $E_{i}$ and a bond energy $V_{i j}$ :

$$
V_{i j}=f_{C}\left(r_{i j}\right)\left[f_{R}\left(r_{i j}\right)+b_{i j} f_{A}\left(r_{i j}\right)\right] .
$$

The indices $i$ and $j$ run over the atoms of the system, and $r_{i j}$ is the distance from atom $i$ to $j$. The function $f_{R}$ represents a repulsive pair potential, which includes the orthogonalization energy when atomic wave functions overlap, and $f_{A}$ represents an attractive pair potential associated with bonding:

$$
\begin{gathered}
f_{R}\left(r_{i j}\right)=A \exp \left(-\lambda_{1} r_{i j}\right), \\
f_{A}\left(r_{i j}\right)=-B \exp \left(-\lambda_{2} r_{i j}\right) .
\end{gathered}
$$

The extra term $f_{C}$ is merely a smooth cut-off function, to limit the range of the potential, since for many applications short ranged functions permit a tremendous reduction in computational effort. 
Here the cut-off function is simply taken as

$$
\begin{aligned}
& f_{C}\left(r_{i j}\right) \\
& \quad= \begin{cases}1, & r_{i j}<R-D, \\
\frac{1}{2}-\frac{1}{2} \sin \left[\frac{(\pi / 2)\left(r_{i j}-R\right)}{D}\right], & R-D<r_{i j}<R+D, \\
0, & r_{i j}>R+D,\end{cases}
\end{aligned}
$$

where $b_{i j}$ represents a measure of the bond order and is for now assumed to be a monotonically decreasing function of the coordination of atoms $i$ and $j$. Details of $b_{i j}$ refer to Tersoff [10].

3.2. Rebo Potential [11]. The binding energy for the hydrocarbon potential is given as a sum over bounds as

$$
E^{\mathrm{REBO}}=\sum_{i} \sum_{j(>i)}\left[V_{R}\left(r_{i j}\right)-b_{i j} V_{A}\left(r_{i j}\right)\right]
$$

where the repulsive and attractive pair terms are given by

$$
\begin{gathered}
V_{R}\left(r_{i j}\right)=f^{C}\left(r_{i j}\right) V_{o}^{R}\left(r_{i j}\right), \\
V_{A}\left(r_{i j}\right)=f^{C}\left(r_{i j}\right) V_{o}^{A}\left(r_{i j}\right), \\
= \begin{cases}f^{C}\left(r_{i j}\right) & r_{i j}<D_{\min }, \\
{\left[\frac{1}{2}+\frac{1}{2} \cos \left(\frac{\pi\left(r_{i j}-D_{\min }\right)}{D_{\max }-D_{\min }}\right)\right],} & D_{\min }<r_{i j}<D_{\max }, \\
0, & r_{i j}>D_{\max } .\end{cases}
\end{gathered}
$$

Here, $r_{i j}$ is the distance between $i$ th and $j$ th atoms, $b_{i j}$ is a bond-order term, and $V_{o}^{R}$ and $V_{o}^{A}$ are functions of $r_{i j}$. For more details of these terms, refer to Brenner et al. [10, 11].

3.3. Airebo Potential [29]. Stuart et al. [29] developed a reactive potential for hydrocarbons with intermolecular interactions. With the adaptive treatment of dispersion, intermolecular repulsion, and torsional interactions, the entire energy is given by the expression

$$
E^{\mathrm{AIREBO}}=\frac{1}{2} \sum_{i} \sum_{j \neq i}\left[E_{i j}^{\mathrm{REBO}}+E_{i j}^{L J}+\sum_{k \neq i, j} \sum_{l \neq i, j, k} E_{k i j l}^{\mathrm{tors}}\right] .
$$

The REBO interaction is based on the form proposed by Tersoff [10]:

$$
E_{i j}^{\mathrm{REBO}}=V_{i j}^{R}+b_{i j} V_{i j}^{A}
$$

in which repulsive and attractive contributions are combined in a ratio determined by the bonding term $b_{i j}$.
The repulsive term has the form used by Brenner et al. $[11,31]$ :

$$
V_{i j}^{R}=w_{i j}\left(r_{i j}\right)\left[1+\frac{Q_{i j}}{r_{i j}}\right] A_{i j} e^{-\alpha_{i j} r_{i j}},
$$

where the parameters $Q_{i j}, A_{i j}$, and $\alpha_{i j}$ depend on the atom types $i$ and $j$. Values for these and all other potential parameters are given in Table II in [29]. The $w_{i j}$ term is a bond-weighing factor,

$$
w_{i j}\left(r_{i j}\right)=S^{t}\left(t_{c}\left(r_{i j}\right)\right),
$$

which switches off the REBO interactions when the atom pairs exceed typical bonding distances. The switching function takes the form

$$
S^{t}(t)=\Theta(-t)+\Theta(t) \Theta(1-t) \frac{1}{2}[1+\cos (\pi t)],
$$

where the switching region for each type of bond is given by a scaling function

$$
t_{c}\left(r_{i j}\right)=\frac{r_{i j}-r_{i j}^{\min }}{r_{i j}^{\max }-r_{i j}^{\min }} .
$$

The attractive pair interaction in (8) is given by a triple exponential,

$$
V_{i j}^{A}=-w_{i j}\left(r_{i j}\right) \sum_{n=1}^{3} B_{i j}^{(n)} e^{-\beta_{i j}^{(n)} r_{i j}},
$$

which is switched off smoothly for non-short-ranged interactions through the use of bond weight. The $b_{i j}$ term in (8) specifies the bond order for the interaction between $i$ and $j$ :

$$
b_{i j}=\frac{1}{2}\left[p_{i j}^{\sigma \pi}+p_{j i}^{\sigma \pi}\right]+\pi_{i j}^{r c}+\pi_{i j}^{d h} .
$$

This term is only roughly equivalent to the usual chemical concept of a bond order and is simply a means of modifying the strength of a bond due to changes in the local environment.

The principal contribution to $b_{i j}$ is the covalent bond interaction, given by the terms $P_{i j}^{\sigma \pi}$ and $P_{j i}^{\sigma \pi}$ :

$$
\begin{aligned}
& P_{i j}^{\sigma \pi}=\left[1+\sum_{k \neq i, j} w_{i k}\left(r_{i k}\right) g_{i}\left(\cos \theta_{j i k}\right) e^{\lambda_{j i k}}+P_{i j}\right]^{-1 / 2}, \\
& P_{j i}^{\sigma \pi}=\left[1+\sum_{k \neq j, i} w_{j k}\left(r_{j k}\right) g_{j}\left(\cos \theta_{i j k}\right) e^{\lambda_{i j k}}+P_{j i}\right]^{-1 / 2} .
\end{aligned}
$$

The penalty function $g_{i}$ imposes a cost on bonds that are too close to one another. Its functional form is a fifth-order spline. When the central atom is a carbon, the spline also depends on the local coordination number, defined as the sum of the 
carbon-only and hydrogen-only coordination numbers. $P_{i j}$ is a function of $N_{i j}^{\mathrm{C}}$ and $N_{i j}^{\mathrm{H}}$.

$$
N_{i j}=N_{i j}^{\mathrm{C}}+N_{i j}^{\mathrm{H}}
$$

$$
\text { where } N_{i j}^{C}=\left(\sum_{k \neq i} \delta_{k C} w_{i k}\left(r_{i k}\right)\right)-\delta_{j C} w_{i j}\left(r_{i j}\right)
$$

counts a carbon-only coordination number, with $\delta_{i j}$ representing a Kronecker delta. The hydrogen-only coordination number $N_{i j}^{\mathrm{H}}$ is defined similarly. Using this coordination number, the angle-bending penalty function $g_{i}$ switches smoothly between a form $g_{\mathrm{C}}^{(1)}$ appropriate for covalent compounds with low coordination and another form $g_{\mathrm{C}}^{(2)}$ suitable for highly coordinated bulk materials:

$$
\begin{aligned}
g_{\mathrm{C}}\left(\cos \theta_{j i k}\right)= & g_{\mathrm{C}}^{(1)}\left(\cos \theta_{j i k}\right)+S^{t}\left(t_{N}\left(N_{i j}\right)\right) \\
& \times\left[g_{\mathrm{C}}^{(2)}\left(\cos \theta_{j i k}\right)-g_{\mathrm{C}}^{(1)}\left(\cos \theta_{j i k}\right) .\right.
\end{aligned}
$$

At intermediate value of $N$, the switching function $S^{t}\left(t_{N}\right)$ provides for a smooth transition, with $S^{t}$ given by (11) and the scaling function $t_{N}$ given by

$$
t_{N}\left(N_{i j}\right)=\frac{N_{i j}-N_{i j}^{\min }}{N_{i j}^{\max }-N_{i j}^{\min }} .
$$

The $e^{\lambda_{j i k}}$ term is added to improve the potential energy surface for abstraction of hydrogen atoms from hydrocarbons, with

$$
\begin{aligned}
\lambda_{j i k}=4 \delta_{i \mathrm{H}}[ & \left(\delta_{k \mathrm{H}} \rho_{\mathrm{HH}}+\delta_{k \mathrm{C}} \rho_{\mathrm{CH}}-r_{i k}\right) \\
& \left.-\left(\delta_{j \mathrm{H}} \rho_{\mathrm{HH}}+\delta_{j \mathrm{C}} \rho_{\mathrm{CH}}-r_{i j}\right)\right],
\end{aligned}
$$

where $\delta_{i j}$ represents the Kronecker delta for atom types $i$ and $j$. The $P_{i j}$ term is a two-dimensional cubic spline [32] in $N_{i j}^{\mathrm{C}}$ and $N_{i j}^{\mathrm{H}}$, whose coefficients are chosen to reproduce the values of $P_{i j}$. These terms are included in the REBO model to give accurate bond energies for small hydrocarbons. Although most REBO parameters were not modified in developing the AIREBO model, the $P_{i j}$ values were modified at two points to counteract the additional torsion energies in the AIREBO potential for unsaturated systems such as ethylene and graphite. In addition to the bonding interactions given by (15), the REBO potential also includes contributions to the bond order from radial and conjugation effects. These enter the potential through the $\pi_{i j}^{r c}$ term, which is a threedimensional cubic spline in the variables $N_{i j}, N_{j i}$, and $N_{i j}^{\text {conj }}$. The indices $N_{i j}$ and $N_{j i}$ are the coordination numbers defined in (16), and $N_{i j}^{\text {conj }}$ is a local measure of conjugation in the $i-j$ bond:

$$
\begin{aligned}
N_{i j}^{\mathrm{conj}}=1 & +\left[\sum_{k \neq i, j} \delta_{k \mathrm{C}} w_{i k}\left(r_{i k}\right) S^{t}\left(t_{\text {conj }}\left(N_{k i}\right)\right)\right]^{2} \\
& +\left[\sum_{l \neq i, j} \delta_{l \mathrm{C}} w_{j l}\left(r_{j l}\right) S^{t}\left(t_{\text {conj }}\left(N_{l j}\right)\right)\right]^{2},
\end{aligned}
$$

with $t_{\text {conj }}$ specifying the range of coordination numbers under which a bond is assumed to be part of a radical or conjugated network:

$$
\begin{aligned}
& t_{\text {conj }}(N)=\frac{N-N^{\mathrm{min}}}{N^{\mathrm{max}}-N^{\mathrm{min}}}, \\
& \pi_{i j}^{r c}=F_{i j}\left(N_{i j}, N_{j i}, N_{i j}^{\mathrm{conj}}\right) .
\end{aligned}
$$

The remaining contribution to the bond-order $b_{i j}$ is $\pi_{i j}^{d h}$ :

$$
\begin{aligned}
\pi_{i j}^{d h}= & T_{i j}\left(N_{i j}, N_{j i}, N_{i j}^{\text {conj }}\right) \\
& \times \sum_{k \neq i, j} \sum_{l \neq i, j}\left(1-\cos ^{2} \omega_{k i j l}\right) \times w_{i k}^{t}\left(r_{i k}\right) w_{j l}^{t}\left(r_{j l}\right) \Theta \\
& \times\left(\sin \left(\theta_{j i k}\right)-s^{\min }\right) \times \Theta\left(\sin \left(\theta_{i j l}\right)-s^{\min }\right), \\
& \cos \omega_{k i j l}=\frac{r_{j i} \times r_{i k}}{\left|r_{j i} \times r_{i k}\right|} \cdot \frac{r_{i j} \times r_{j l}}{\left|r_{i j} \times r_{j l}\right|} .
\end{aligned}
$$

The bond-weighting function is

$$
w_{t}^{\prime}\left(r_{i j}\right)=S^{\prime}\left(t_{c}^{\prime}\left(r_{i j}\right)\right)
$$

The scaling function $t_{c}^{\prime}$ is

$$
t_{c}^{\prime}\left(r_{i j}\right)=\frac{r_{i j}-r_{i j}^{\min }}{r_{i j}^{\max }-r_{i j}^{\min }}
$$

The $L J$ contribution to the $i-j$ pair energy is

$$
\begin{aligned}
E_{i j}^{L J}= & S\left(t_{r}\left(r_{i j}\right)\right) S\left(t_{b}\left(b_{i j}^{*}\right)\right) C_{i j} V_{i j}^{L J}\left(r_{i j}\right) \\
& +\left[1-S\left(t_{r}\left(r_{i j}\right)\right)\right] C_{i j} V_{i j}^{L J}\left(r_{i j}\right),
\end{aligned}
$$

includes the traditional $L J$ term

$$
V_{i j}^{L J}\left(r_{i j}\right)=4 \varepsilon_{i j}\left[\left(\frac{\sigma_{i j}}{r_{i j}}\right)^{12}-\left(\frac{\sigma_{i j}}{r_{i j}}\right)^{6}\right]
$$

modified by several sets of switching functions. The switching function $S(t)$ is

$$
S(t)=\Theta(-t)+\Theta(t) \Theta(1-t)\left[1-t^{2}(3-2 t)\right]
$$

Both have continuous first derivatives at the switching region boundaries.

Below a certain separation distance $r_{i j}^{L J}$ max , the magnitude of the $L J$ term depends on the bonding environment. The gradual exclusion of $L J$ interactions as $r_{i j}$ changes is controlled by the $t_{r}$, scaling function,

$$
t_{r}\left(r_{i j}\right)=\frac{r_{i j}-r_{i j}^{L J \min }}{r_{i j}^{L J \max }-r_{i j}^{L J \min }} .
$$


At intermolecular distances, the $L J$ interaction is included only if there is no significant bonding interaction between two atoms, as specified by $t_{b}$ switch,

$$
t_{b}\left(b_{i j}\right)=\frac{b_{i j}-b_{i j}^{\min }}{b_{i j}^{\max }-b_{i j}^{\min }}
$$

And if the atoms $i$ and $j$ are not connected by two or fewer intermediate atoms, this latter switch is controlled by bond weights,

$$
\begin{aligned}
C_{i j}=1-\max \left\{w_{i j}\left(r_{i j}\right), w_{i k}\left(r_{i k}\right) w_{k j}\left(r_{k j}\right),\right. \\
\left.\forall k w_{i k}\left(r_{i k}\right) w_{k l}\left(r_{k l}\right) w_{l j}\left(r_{l j}\right), \forall k, l\right\} .
\end{aligned}
$$

The torsional potential for the dihedral angle determined by atoms $i, j, k$, and $l$ is controlled by the term

$$
E_{k i j l}^{\mathrm{tors}}=w_{k l}\left(r_{k l}\right) w_{i j}\left(r_{i j}\right) w_{j l}\left(r_{j l}\right) V^{\mathrm{tors}}\left(\omega_{k i j l}\right)
$$

where

$$
V^{\mathrm{tors}}\left(\omega_{k i j l}\right)=\frac{256}{405} \varepsilon_{k i j l} \cos ^{10}\left(\frac{\omega_{k i j l}}{2}\right)-\frac{1}{10} \varepsilon_{k i j l} .
$$

\section{Results and Discussion}

At different strain rates we simulate CNTs subjected to a uniform axial loading at its both ends using Tersoff potential [10], Rebo potential [11], and Airebo potential [29] to understand the effect of strain rates on their potential energy and elastic, elastic-plastic properties. To understand the effect of Van der Waals interaction and torsion with bond-order potential per atom potential energy with strain of singlewalled 12-12 CNTs subjected to a uniform tension at their both ends obtained by different potentials are compared to each other. At high strain rate, we simulate CNTs subjected to an axial tension at their both ends using five different potentials such as Tersoff potential [10], Rebo potential [11], Rebo potential [31], Inclusion $L J$ with Rebo potential [11], and Airebo potential [29] to study the effect of potentials on their elastic, elastic-plastic properties. Tersoff potential [10] and Rebo potential [11] are bond-order potential by short range cut-off functions. Out of the cut-off distance, carbon atoms in CNT in different planes and layers do not interact although they experience weak Van der Waals interaction. For nonbonded atoms, although the Van der Waals attraction and repulsion exhibit at a short distance but for the bonded atoms, these Van der Waals attraction and repulsion occur at a long distance. When bond-order potential is used, torsional term must be included to get higher accuracy of the solution because during simulation position of atoms changes due to interacting forces among bonded atoms and bond experiences some angular displacement. Stuart et al. [29] introduced long range Van der Waals and torsional terms in Rebo potential [11] and obtained elastic constants, bond energy, vacancy formation energy, enthalpy of carbon in diamond, and graphite structures using Rebo potential [11] and modified Rebo potential, that is, Airebo potential [29], and compared their results obtained by Airebo potential [29] and Rebo potential [11] with experimental results. The results obtained by Airebo potential [29] show better agreement than that of Rebo potential [11] with experimental results. From the study of diamond and graphite structure by Stuart et al. [29], it is clear that Airebo potential [29] is more suitable for getting highly accurate solution of $\mathrm{C}, \mathrm{H}$ structures than that of Rebo potential [11]. Firstly we obtain per atom potential energy of a single-walled $12-12 \mathrm{CNT}$ as a function of axial strain at a very low strain rate using three different potentials such as Airebo potential [29], Rebo potential [11], and Tersoff potential [10] to understand the effect of Van der Waals interaction and torsional effects on bondorder potential whose effects are reflected in the elastic, elastic-plastic properties of CNTs. We obtain the stress strain relationship of a single-walled $12-12 \mathrm{CNT}$ subjected to a uniaxial loading by molecular dynamics simulation using three different potentials at four different strain rates, that is, from very low strain rate to high strain rate, and compare these with that obtained by Belytschko et al. [30] to get reliable potential for studying the mechanics of CNTs. Effects of strain rates on the elastic, elastic-plastic properties of a single-walled 12-12 CNT subjected to an axial loading using different potentials are investigated. For very low to high strain rates, a suitable potential is selected for the study of nanomechanics of CNTs from the comparative study of per atom potential energy and tensile stress as a function of axial strain using three different potentials with those of published results. To understand the nanomechanics of single-walled, double-walled, three-walled, and four-walled CNTs at high strain rate, five different potentials are also used which are Rebo potential [11], Rebo potential [31], Airebo potential [29], Tersoff potential [10], and Inclusion $L J$ in Rebo potential [11]. In Rebo potential [11] only bond-order term is included. In Rebo potential [31] torsion term is added with bond-order term. In Airebo potential [29] Van der Waals interaction and torsional terms are added with bond-order term. In Tersoff potential [10], only bond order is added. In Inclusion $L J$ in Rebo potential [11], Van der Waals interaction is added with bond-order term. Effects of layering and aspect ratio on the elastic, elastic-plastic properties of CNTs are studied at high strain rate considering five different potentials as comparative manners with those of published results. We compare the elastic, elastic-plastic properties of different types of CNTs subjected to a tensile load at its opposite end at a high strain rate using five different potentials with that of Liew et al. [13]. Young's modulus of CNTs is obtained from the slope of the stress strain relationship of CNTs till 5\% strain. Yield strength and yield strain of CNTs are measured from a point of the stress strain curve where its slope is the lowest. Ultimate strength and ultimate strain of CNTs are measured from a point of stress strain curve where the stress is the highest. Poisson's ratio of CNTs is calculated considering the change of its diameter and length just before fracture using the ratio of lateral to longitudinal strain. Besides, we present the elastic, elastic-plastic properties of CNTs at high strain rate using different potentials with the published results as a tabular form. We also obtain Young's modulus, yield strength, yield strain, ultimate strength, and ultimate strain of single-walled, 


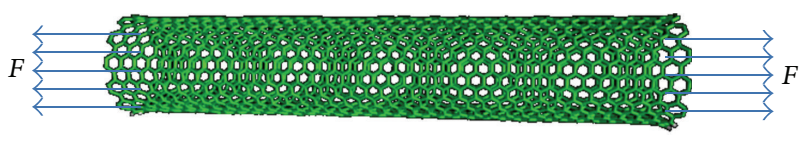

(a)

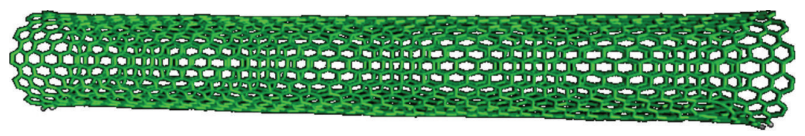

(b)

FIGURE 1: Physical configuration of a single-walled $(12,12) \mathrm{CNT}$ at length-to-diameter ratio, 4.51: (a) original configuration; (b) snapshot just before fracture obtained by molecular dynamics simulation using Airebo potential [29].

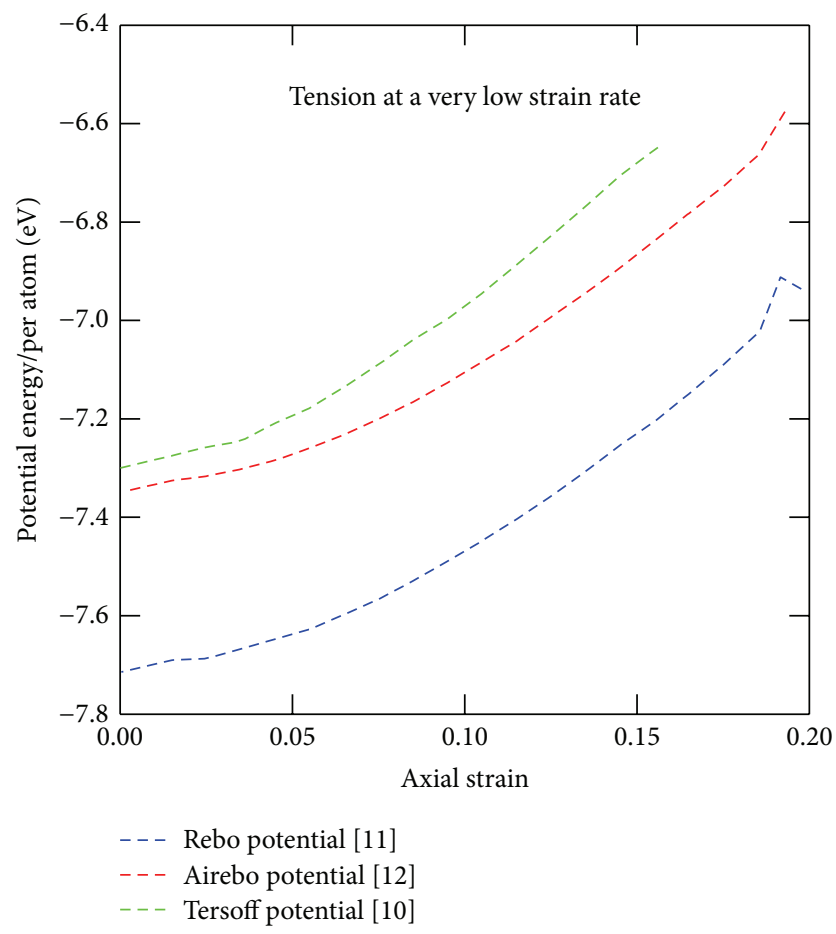

FIGURE 2: Per atom potential energy of a single-walled $(12,12)$ CNT with length-to-diameter ratio 9.1 as a function of axial strain when both ends of CNT are stretched at a velocity $0.09 \mathrm{~m} / \mathrm{s}$ (with very low strain rate).

double-walled, three-walled, and four-walled CNTs from the axial stress strain relationships of different types of CNTs using different potentials by molecular dynamics simulation and present in tabular form.

Figure 1 presents the original and final physical configuration just before breaking of a single-walled 12-12 CNT having length-to-diameter ratio 4.51 using Airebo potential [29]. Figure 2 illustrates per atom potential energy of a singlewalled 12-12 CNT with aspect ratio 9.1 as a function of strain at a very low strain rate (stretching velocity at both ends of a CNT is $0.09 \mathrm{~m} / \mathrm{s}$ ) obtained by Rebo potential [11], Airebo potential [29], and Tersoff potential [10] as a comparative manner. From Figure 2, it is clearly observed that per atom potential energy of CNTs obtained by Tersoff potential is the lowest, per atom potential energy of CNTs obtained by Rebo potential [11] is the highest, and per atom potential energy obtained by Airebo potential [29] remains in between them due to addition of Van der Waals and torsional interactions with bond-order term in Airebo potential [29]. Although the slope of per atom potential energy as a function of strain for Rebo potential [11] and Airebo potential [29] is almost the same, the slope of per atom potential energy as a function of axial strain of single-walled 12-12 CNTs obtained by Tersoff potential [10] is higher than that of Rebo potential [11] and Airebo potential [29]. From Figure 2, it is observed that fracture strain of a single-walled 12-12 CNT obtained by Rebo potential [11] and Airebo potential [29] is almost the same, but fracture strain of single-walled 12-12 CNTs obtained by Tersoff potential [10] is the lowest.

Figure 3 illustrates the comparative study of the tensile stress strain relationship of single-walled 12-12 CNTs subjected to an axial tension using Rebo potential [11], Airebo potential [29], and Tersoff potential [10] at four different strain rates with that of Belytschko et al. [30]. From Figure 3(a), it is observed that the tensile stress strain relationships of single-walled 12-12 CNTs obtained by Rebo potential [11] and Airebo potential [29] at a very small strain rate (stretching velocity of both ends of CNTs is $0.09 \mathrm{~m} / \mathrm{s}$ ) are very close to that of single-walled 12-12 CNTs by Belytschko et al. [30] but the tensile stress strain relationship obtained by Tersoff potential [10] deviates a large extent from that of Belytschko et al. [30]. From Figure 3(b), it is observed that tensile stress strain relationship of a single-walled 12 12 CNT obtained by Rebo potential [11] and Airebo potential [29] at a small strain rate (stretching velocity at both ends of CNTs is $0.5 \mathrm{~m} / \mathrm{s}$ ) is almost the same and up to yield strength and the tensile stress strain relationship obtained by Rebo potential [11] and Airebo potential [29] matches with that of Belytschko et al. [30] but after yielding, the tensile stress strain relationship of a single-walled 12-12 CNT obtained by Rebo potential [11] and Airebo potential [29] disagrees with that of Belytschko et al. [30]. At a very low (stretching velocity at both ends of CNTs is $0.09 \mathrm{~m} / \mathrm{s}$ ) and low strain (stretching velocity at both ends of CNTs is $0.5 \mathrm{~m} / \mathrm{s}$ ) rate, the slope of stress strain relationship obtained by Tersoff potential is higher than that of Rebo potential [11], Airebo potential [29], and Belytschko et al. [30] because decreasing rate of per atom potential energy of a single-walled 12-12 CNT with axial strain obtained by Tersoff potential is higher than that of Rebo potential [11] and Airebo potential [29] as shown in Figure 2. Ultimate stress and strain of single-walled 1212 CNT obtained by Rebo potential [11] and Airebo potential [29] are higher than that of Belyschko et al. [30] when the stretching velocity at both ends of CNTs is $0.5 \mathrm{~m} / \mathrm{s}$. From Figure 3(c), it is observed that ultimate strength and strain of a single-walled 12-12 CNT at moderate strain rate (stretching velocity at both ends of CNTs is $5 \mathrm{~m} / \mathrm{s}$ ) obtained by Rebo potential [11] are higher than those of Airebo potential [29] because at moderate strain rate Van der Waals and torsional effect decrease per atom potential energy of CNTs with strain for which it takes less strain and time to reach the lowest per atom potential energy for fracture but for fracture of 
CNTs, opposite phenomenon is observed when tension test of a CNT is carried out using Rebo potential [11] at moderate strain rate. At high strain rate the deviation between ultimate strength and strain of single-walled 12-12 CNTs obtained by Rebo potential [11] and Airebo potential [29] is higher than that of moderate strain rate. At moderate and high strain rate ultimate strength of single-walled 12-12 CNTs obtained by Airebo potential [29] is closer to that of Belytschko et al. [30] than that of Rebo potential [11]. In all strain rates, the slope of the stress strain relationship of a singlewalled 12-12 CNT obtained by Tersoff potential [10] is higher than that of Rebo potential [11], Airebo potential [29], and Belytschko et al. [30]. As at very low strain rate the stress strain relationship of a single-walled 12-12 CNT subjected to axial loading obtained by Rebo potential [11] and Airebo potential [29] is very close to that of Belytschko et al. [30], Airebo potential [29] and Rebo potential [11] are suitable for the study of nanomechanics of CNTs at a very low strain rate. At a very low strain rate per atom potential energy with strain of a single-walled 12-12 CNT obtained Airebo potential [29] is more accurate than that of Rebo potential [11] because Stuart et al. [29] observed that the vacancy formation energy, bond energy, and enthalpy of graphite and diamond by Airebo potential [29] is closer to those of experimental result than those obtained by Rebo potential [11]. At moderate and high strain rates Airebo potential [29] will give more accurate per atom potential energy and elastic, elastic-plastic response of CNTs during loading till fracture than those of other potentials because each bonded atom experiences Van der Waals interaction and torsional effect during loading and these two terms are not included in the rest of other potentials together.

Figure 4 describes the stress strain relationship of a single-walled 12-12 CNT having length-to-diameter ratio, 9.1, subjected to uniform tension at its opposite ends using Airebo potential [29] by molecular dynamics simulation at high strain rate (stretching velocity at both ends of CNTs is $20 \mathrm{~m} / \mathrm{s}$ ). The stress strain relationship curve gives three onset cut-off diameters, where the elastic, elastic-plastic properties change suddenly. In Figure 4, the slope of the curve at a certain range is constant; that is, the stiffness of the CNT remains constant and then the slope of the stress strain relationships of the CNT gradually decreases to a zero value; that is, the stiffness of the CNT gradually decreases and when the diameter of the CNT suddenly drops, the slope of the stress strain curve becomes zero and this is the phase change of CNT due to strain hardening and decreasing its diameter suddenly enhances its stiffness to a greater extent till fracture.

Figures 5(a) and 5(b) illustrate the effect of different potentials on per atom potential energy and per atom total energy of CNTs as a function of strain with the tensile load at their both ends by molecular dynamic simulation at high strain rate. Per atom potential energy of loaded CNTs at a specific strain before fracture obtained by Rebo potential [11] is higher than that obtained by Airebo potential [29] as shown in Figure 5(a) due to the absence of the torsional and Van der Waals interaction in Rebo potential [11] and the fracture strain of CNTs obtained by Rebo potential [11] is higher than that of Airebo potential [29] although the per atom potential energy of CNTs during fracture obtained by Rebo potential [11] and Airebo potential [29] is almost the same. As the slope of per atom potential energy as a function of axial strain of loaded CNTs obtained by Rebo potential [11] and Airebo potential [29] is nearly the same and fracture strain of CNTs obtained by Rebo potential [11] considering per atom potential energy is higher than that of Airebo potential [29], the slope of the stress strain relationship of CNTs obtained by Rebo potential [11] and Airebo potential [29] should be the same and ultimate fracture strength of CNTs obtained by Rebo potential [11] should be higher than that of Airebo potential [29]. Although Tersoff potential [10] is also a bondorder potential like Rebo potential [11], the slope of per atom potential energy of CNTs as a function of strain obtained by Tersoff potential [10] is the highest among those of other potentials which means the slope of the stress strain of loaded CNTs obtained by Tersoff potential [10] should be higher than that of other potentials. From Figure 5(a) it is clear that ultimate strength of CNTs obtained by Tersoff potential [10] should be the lowest of all other potentials. Figure 5(b) illustrates per atom total energy as a function of strain of CNTs obtained by five different potentials and after fracture per atom total energy of CNTs obtained by these potential decreases a little amount due to its increased kinetic energy. Effect of kinetic energy on per atom total energy of CNTs obtained by all of the mentioned potentials is small.

Liew et al. [13] obtained tensile stress strain relationship of single-walled, double-walled, three-walled, and four-walled CNTs with aspect ratios 9.1 and 4.51, respectively, using Rebo potential [11] with Van der Waals interaction without minimizing the initial configuration of CNTs before applying tensile loading at high strain rate (stretching velocity at both ends of the CNTs is $20 \mathrm{~m} / \mathrm{s}$ ). Before loading CNTs, it remains in minimized energy level. So to get accurate elastic, elastic-plastic response of CNTs we should minimize energy before applying loading because after minimization of energy applying potentials in molecular dynamics simulation, the position of $\mathrm{C}$ atoms changes and little changes are observed in shapes of CNTs. Besides, Agrawal et al. [14] studied elastic, elastic-plastic response of single wall CNTs subjected to a uniform axial loading varying $\mathrm{C}-\mathrm{C}$ bond length and bond angle at high strain rate using only bond-order Rebo potential [11]. In the present simulation of CNTs, we minimize different types of CNTs with aspect ratios 9.1 and 4.51, respectively, before loading using five different potentials and then apply load at its both ends (stretching velocity at both ends of CNTs is $20 \mathrm{~m} / \mathrm{s}$ ) like Liew et al. [13] and Agrawal et al. [14]. We compare the tensile stress strain relationships of single-walled CNTs obtained by us using Rebo potential [11], Rebo potential [31], Airebo potential [29], Inclusion $L J$ with Rebo potential [11], and Tersoff potential [10] with those of Agrawal et al. [14] and Liew et al. [13]. Besides, we compare the tensile stress strain relationships of double-walled, three-walled, and four-walled CNTs obtained by us using Rebo potential [11], Rebo potential [31], Airebo potential [29], Inclusion $L J$ with Rebo potential [11], and Tersoff potential [10] with those of Liew et al. [13]. From the comparative studies, we try to obtain accurate tensile stress strain relationships of different types of CNTs and suitable potential for finding 


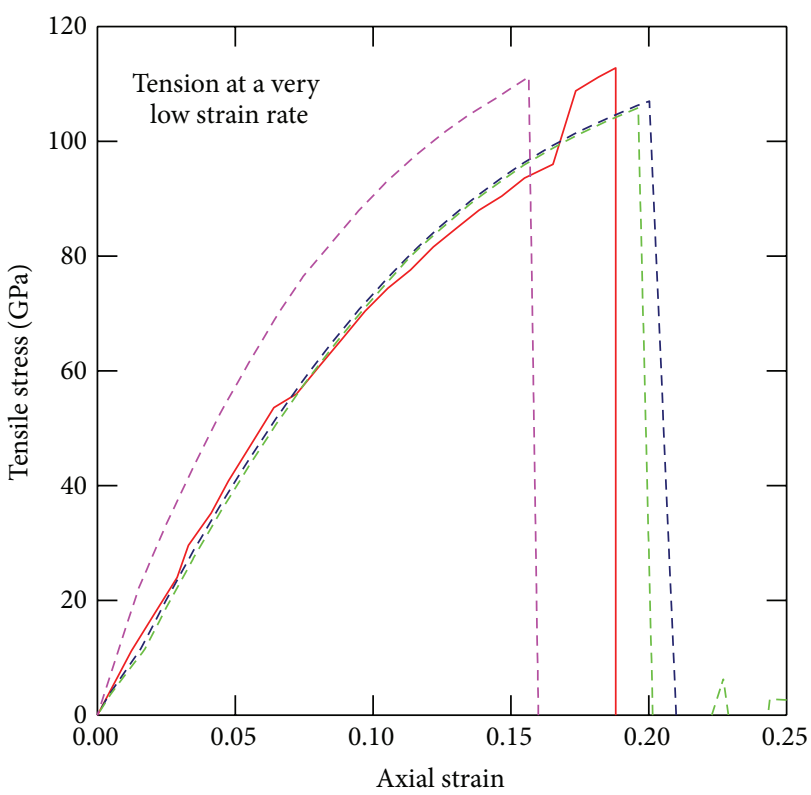

_ Belytschko et al. [16] _- - Rebo potential [11]

- - - Airebo potential [12] _ - - Tersoff potential [10]

(a)

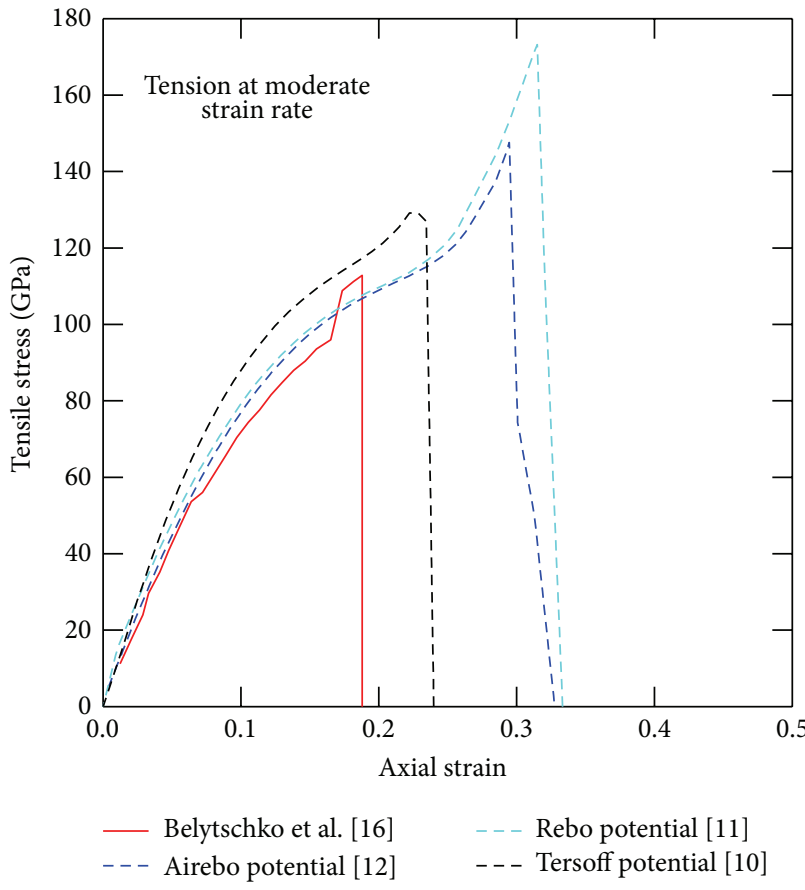

(c)

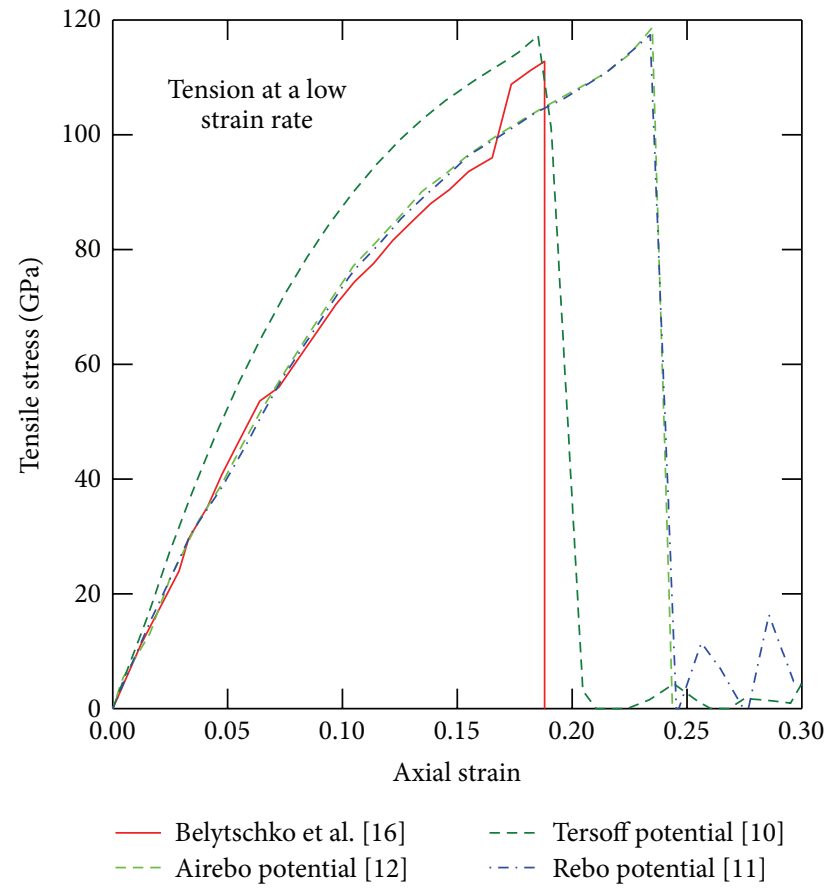

(b)

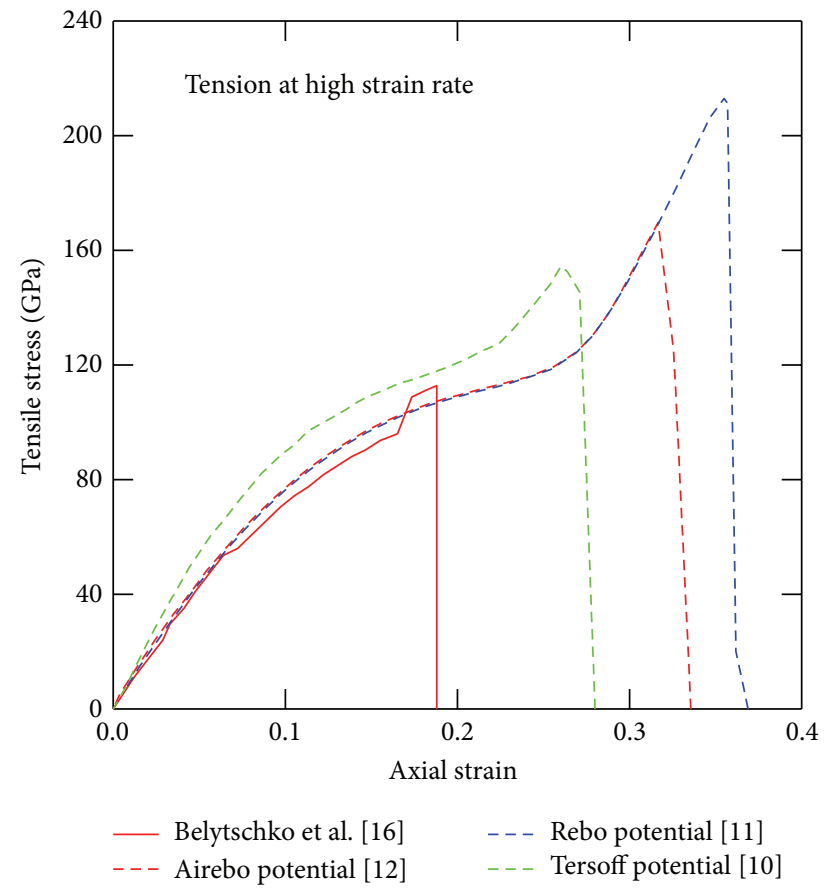

(d)

FIGURE 3: Comparison of the stress strain relationship of a single-walled 12-12 CNT subjected to a uniform tensile load at its both ends for the case of different strain rates using different potentials with those obtained by DFT calculation by Belytschko et al. [30]: (a) at very low strain rate (stretching velocity of both ends of a CNT $=0.09 \mathrm{~m} / \mathrm{s}) ;(\mathrm{b})$ at low strain rate (stretching velocity of both ends of a CNT $=0.5 \mathrm{~m} / \mathrm{s}) ;(\mathrm{c})$ at moderate strain rate (stretching velocity of both ends of CNTs $=5 \mathrm{~m} / \mathrm{s}$ ); (d) at high strain rate (stretching velocity of both ends of a CNT $=$ $20 \mathrm{~m} / \mathrm{s})$. 
TABLE 1: Comparison of some parameters in Airebo potential by Stuart et al. [29] with those of Sinnott et al. [12] which were used by Liew et al. [13] in their simulation of CNTs during coupling Van der Waals interaction with Rebo [11, 31] potentials.

\begin{tabular}{|c|c|c|c|}
\hline $\begin{array}{l}\text { Parameters used for the carbon carbon Van der Waals } \\
\text { interactions (excluding the spline coefficients) }\end{array}$ & Sinnott et al. [12] & Airebo potential Stuart et al. [29] & Units \\
\hline$\varepsilon_{\mathrm{CC}}$ & 0.0042038 & 0.00284 & ev \\
\hline$\varepsilon_{\mathrm{CH}}$ & - & 0.00206 & ev \\
\hline$\varepsilon_{\mathrm{HH}}$ & - & 0.00150 & ev \\
\hline$\sigma_{\mathrm{CC}}$ & 3.37 & 3.4 & $\AA$ \\
\hline$\sigma_{\mathrm{CH}}$ & - & 3.025 & $\AA$ \\
\hline$\sigma_{\mathrm{HH}}$ & - & 2.65 & $\AA$ \\
\hline$r_{\mathrm{CC}}^{L J \min }$ & 2.28 & 3.4 & $\AA$ \\
\hline$r_{\mathrm{CC}}^{L J \text { medium }}$ & 3.40 & - & $\AA$ \\
\hline$r_{\mathrm{CC}}^{L J \max }$ & 10 & 10 & $\AA$ \\
\hline$r_{\mathrm{CH}}^{L J \min }$ & - & 3.025 & $\AA$ \\
\hline$r_{\mathrm{CH}}^{L J \max }$ & - & 3.396228 & $\AA$ \\
\hline$r_{\mathrm{HH}}^{L J \min }$ & - & 2.65 & $\AA$ \\
\hline$r_{\mathrm{HH}}^{L J \max }$ & - & 2.975208 & $\AA$ \\
\hline
\end{tabular}

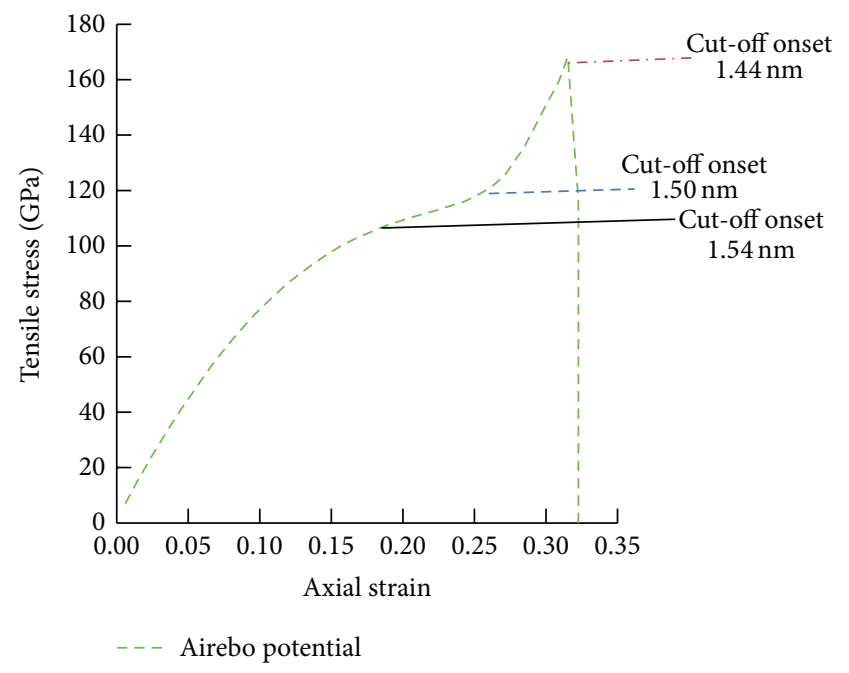

FIgURE 4: Stress strain curves for an ideal single-walled 12-12 CNT having length-to-diameter ratio 9.1 at $300 \mathrm{~K}$ temperature obtained by Airebo potential [29]. The onset of the cut-off function can be perceived as an artificial peak. The flat regime depicts a sudden bond elongation to a length corresponding to the cut-off. After this flat region, the diameter of CNT drops.

more accurate elastic, elastic-plastic response of CNTs during loading at high strain rate. Figures 6(a) and 6(b) illustrate the tensile stress strain relationship of single-walled 1212 CNT having length diameter ratios 9.1 and 4.5, respectively, by five different potentials with two published results as a comparative manner at high strain rate (stretching velocity at both ends of CNTs is $20 \mathrm{~m} / \mathrm{s}$ ). The nature of the elastic-plastic region obtained by Agrawal et al. [14] is totally different from that of Liew et al. [13]. The elastic-plastic region obtained by the present five different potentials agrees well with that of Agrawal et al. [14] although Agrawal et al. [14] solved the
CNT problem using Rebo potential [11]. On the other hand Liew et al. [13] solved the CNT problem using Rebo potential including Van der Waals interactions and we also solved the CNT problem using Airebo potential [29], Inclusion $L J$ with Rebo potential [11] in which Airebo potential [11] consist of long range Van der Waals interaction and torsional effect of bond as well as Inclusion $L J$ with Rebo potential [11] consist of Rebo potential [11] and Van der Waals interaction. The elasticplastic region of CNT obtained by Airebo potential agrees well with that of Agrawal et al. [14] and the ultimate strength of CNT obtained by Agrawalet al. [14] is higher from that of Airebo potential [29] because they did not consider Van der Waals interactions and torsional effect. From the comparative study, it is clear that the elastic, elastic-plastic properties of CNT obtained by Airebo potential [29] are more reliable and accurate. The elastic, elastic-plastic response of CNT by Liew et al. [13] does not agree well with other results due to lack of minimization of CNT structure before loading. Besides, the magnitude of the potential depth for Van der Waals interaction as shown in Table 1 is nearly two times that of ours in Airebo potential [29] and it is another reason to deviate the stress strain relationship of single-walled 12-12 CNTs by Liew et al. [13] from that of ours and Agrawal et al. [14]. Effects of Van der Waals interaction and torsional effect on the per atom potential energy with strain, stress strain relationship of CNTs subjected to uniform tension at high strain rate are clearly observed in Figures 5 and 6. Figures 7(a) and 7(b) illustrate the tensile stress as a function of axial strain of a singlewalled 10-10 CNT with length-to-diameter ratios 9.1 and 4.51, respectively, using five different potentials with the published results by Liew et al. [13]. Up to elastic region, the stress strain relationship obtained by five different potentials agrees well with Liew et al. [13] but in the elastic-plastic region, the shapes of the curves of the stress strain relationship of CNT by us for five different potentials are different from that of Liew et al. [13] which contradicts the reliability of the stress 


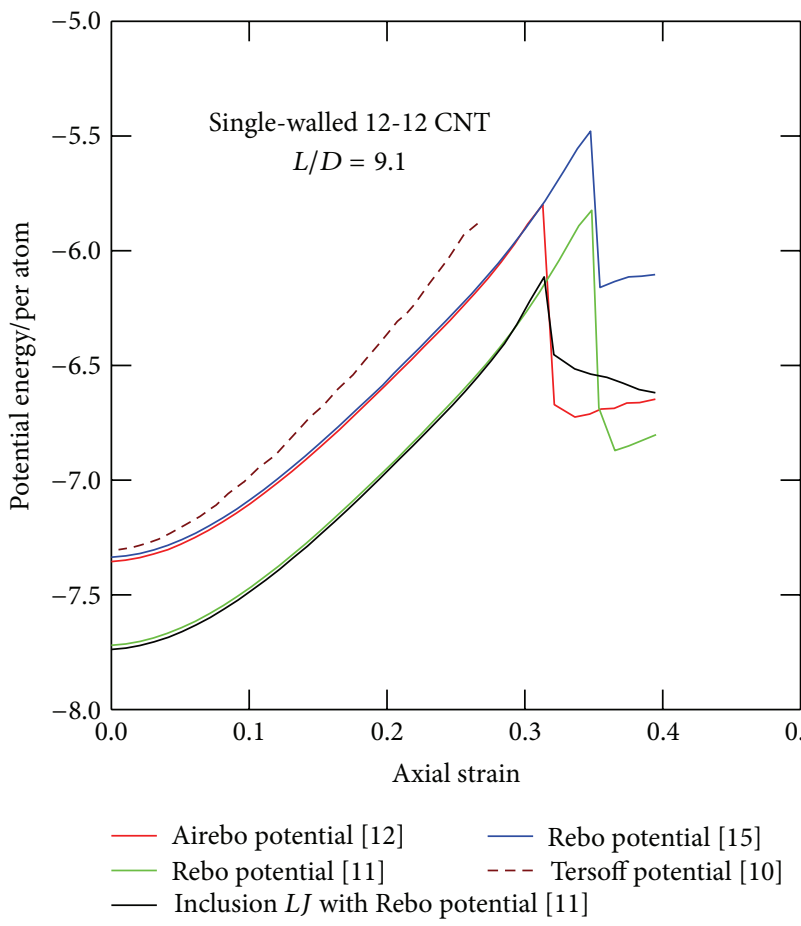

(a)

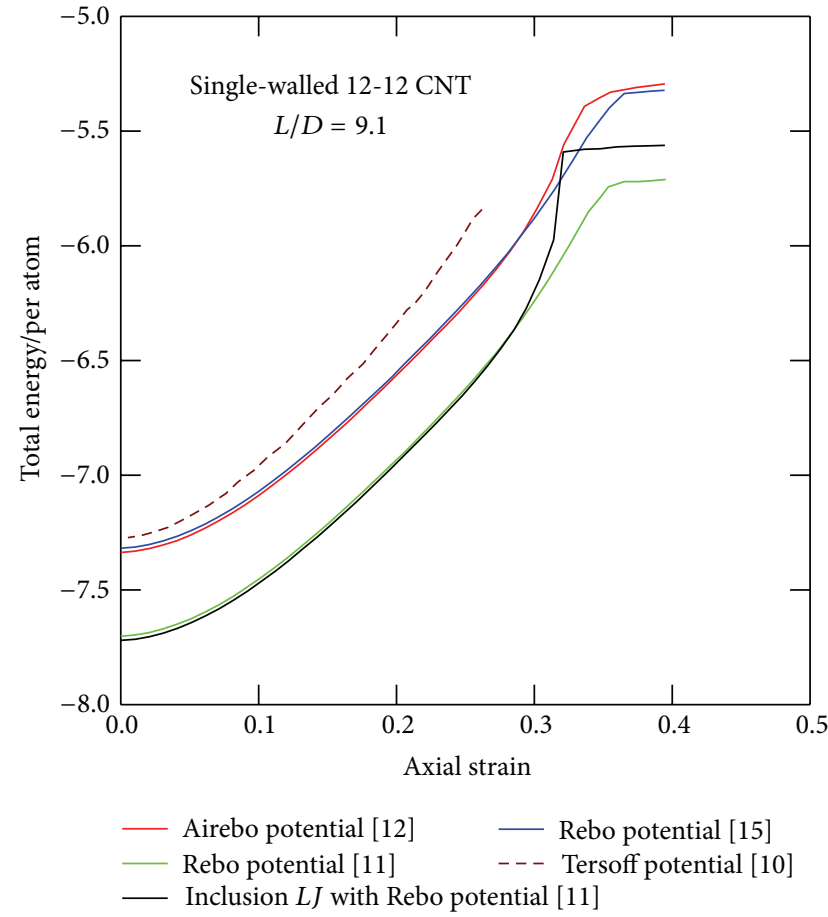

(b)

FIGURE 5: Effect of empirical potentials: (a) on the average potential energy as a function of axial strain; (b) per atom total energy as a function of axial strain of a single-walled 12-12 CNT under tension with steps at $300 \mathrm{~K}$.

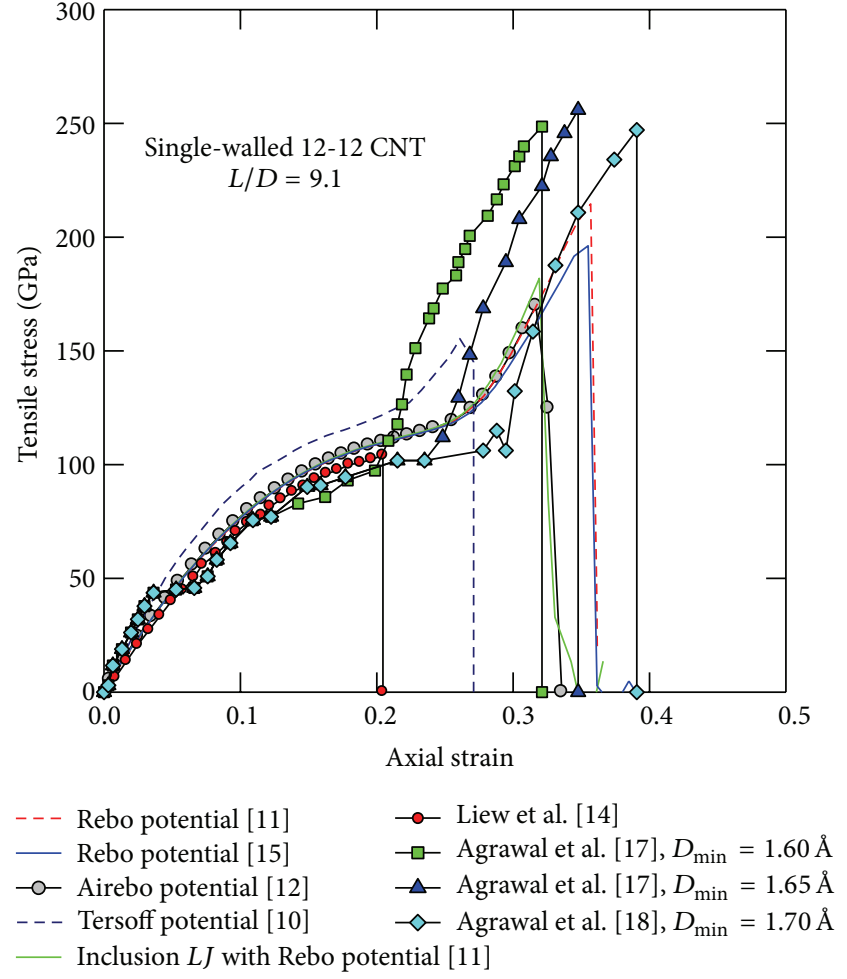

(a)

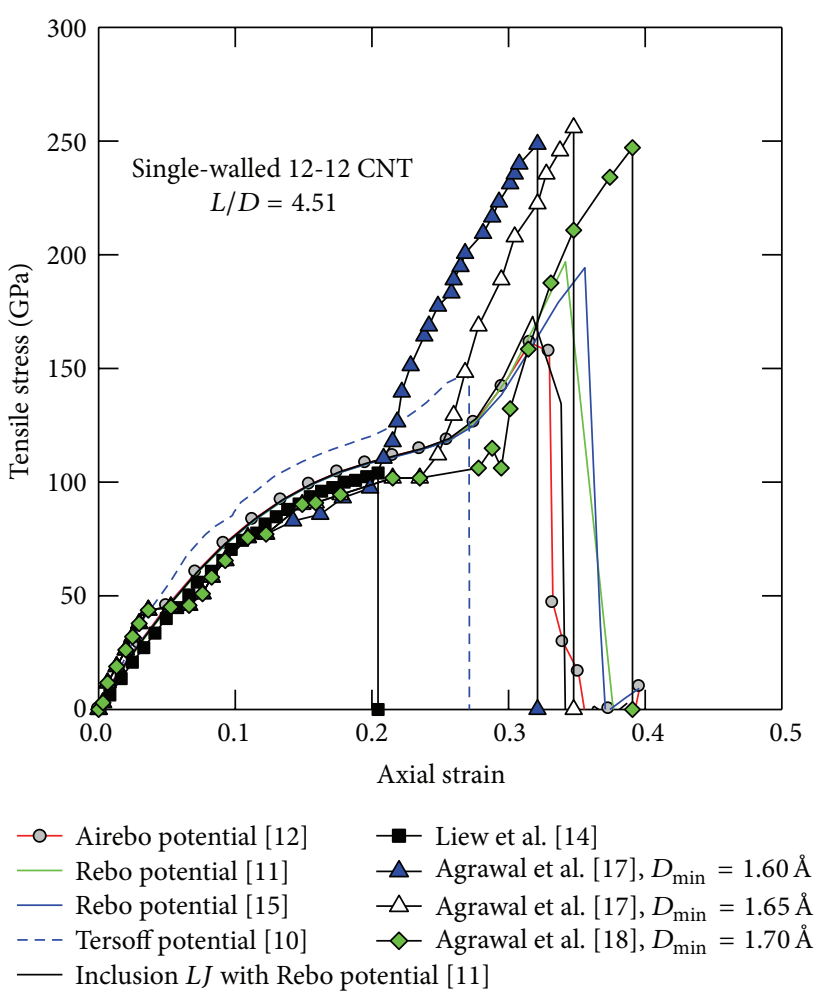

(b)

FIGURE 6: Comparison of the elastic, elastic-plastic response of a single-walled 12-12 CNT using different potentials with the published results: (a) $L / D=9.1$; (b) $L / D=4.51$. 


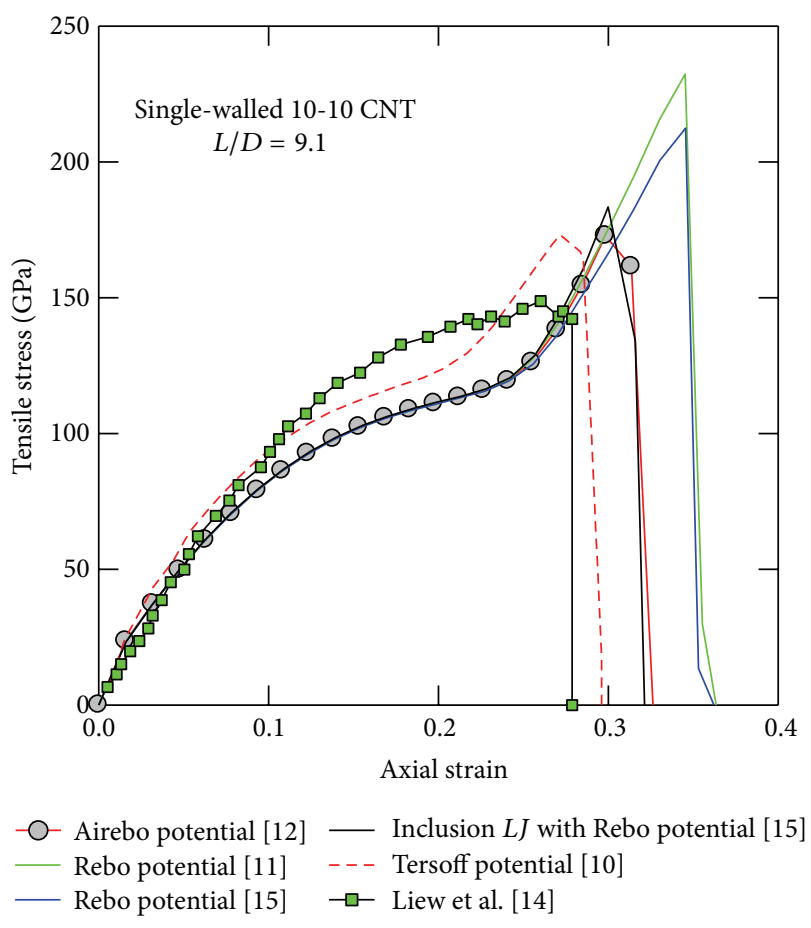

(a)

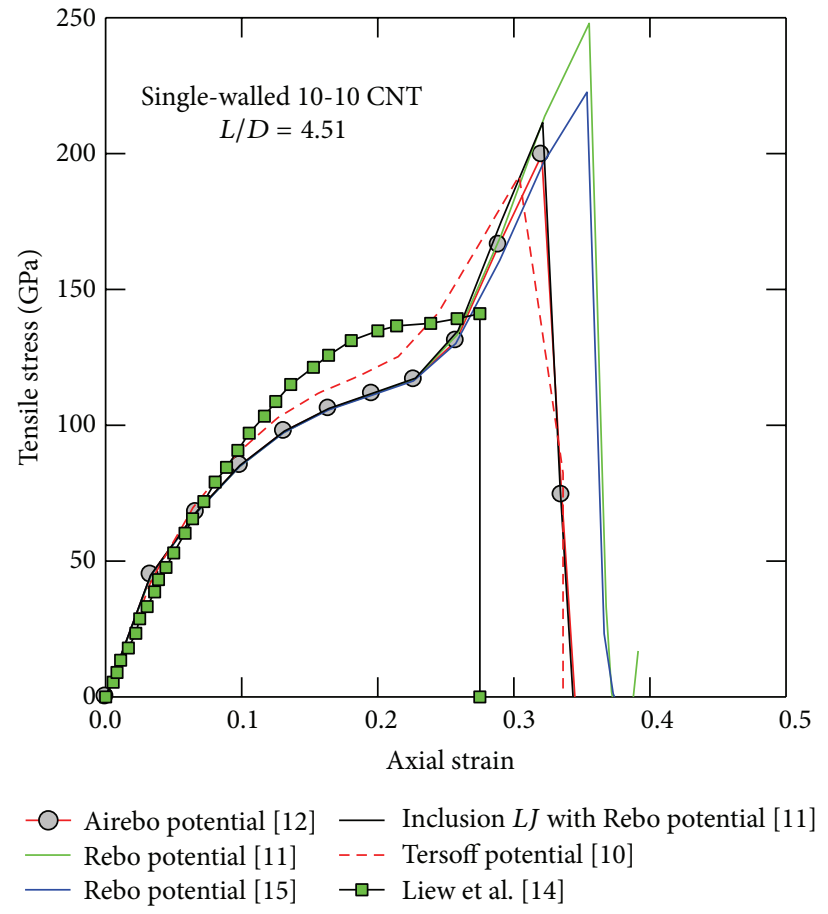

(b)

FIGURE 7: Comparison of the elastic, elastic-plastic response of a single-walled 10-10 CNT using different potentials with the published results: (a) $L / D=9.1$; (b) $L / D=4.51$.

strain relationship obtained by Liew et al. [13] because the stress strain relationship of CNTs for five different potentials shows three onset cut-off which ensure sudden change in diameter of the CNT which is also reflected in the recent published results by Agrawal et al. [14] and Sammalkorpi et al. [21]. Variations of stress strain relationships of single-walled CNTs are observed if length-to-diameter ratio and diameter of CNTs are varied as shown in Figures 6 and 7.

From Figures 8(a) and 8(b), it is observed that the stress strain relationship of a double-walled CNT differs for five different potentials although the trends of the shape of the stress strain relationship are similar and the elastic range, the stress strain relationship obtained by five different potentials are nearly equal to that of Liew et al. [13]. In the elastic-plastic range of double-walled CNTs, our results obtained by five different potentials disagree with that of CNTs obtained by Liew et al. [13]. Figures 9 and 10 illustrate the same stress strain relationship of three-walled and four-walled CNTs in two aspect ratios 9.1 and 4.5 using five different potentials with the published results by Liew et al. [13] as a comparative manner at high strain rate; the stress strain relationship in the elastic range of three-walled and four-walled CNTs obtained by five different potentials agrees with that of Liew et al. [13], but the stress strain relationship in the elastic-plastic range of three-walled and four-walled CNTs obtained by five different potentials does not agree with that of Liew et al. [13]. Among the stress strain relationships obtained by Tersoff potential [10], Rebo potential [11], Rebo potential [31], Inclusion $L J$ with Rebo potential [11], and Airebo potential [29], there is the highest reliability in the stress strain relationship obtained by using Airebo potential [29] because this potential includes long range Van der Waals interaction and torsional effect of bonds with Rebo potential [11]. Naturally every bonded atom experiences weak Van der Waals interaction after a certain distance; when bonded atom displaces its position, there is some angular displacement for which $\mathrm{C}-\mathrm{C}$ bonds of CNTs experience torsion. At very low strain range the stress strain relationship of a single-walled 12-12 CNT obtained by Rebo potential [11] and Airebo potential [29] agrees well with that of Belytschko et al. [30]. Although at very low strain range, the effects of Van der Waals interaction and torsional effect on the elastic, elastic-plastic response are negligible, at moderate and high strain rate, the effects of Van der Waals interaction and torsion effect play important roles which are observed in the graphical results of stress strain relationships of CNTs during loading (see Figures 3 and 6-10). Effects of Van der Waals interaction and torsional effects on per atom potential energy of CNTs during tension at different strain rates are observed (see Figures 2 and 5). Effects of layering on the stress strain relationship of CNTs subjected to tensile load at a high strain rate are also observed as shown in Figures 8 to 10. Effects of Van der Waals interaction and torsion on the stress strain relationships of double-walled, three-walled, and four-walled CNT are observed from their comparative study of those of Rebo potential [11], Rebo potential [31], Inclusion LJ with Rebo potential [11], and Airebo potential [29] as observed in Figures 8 to 10. Figure 11 illustrates the physical configuration of four-walled CNTs at different steps 


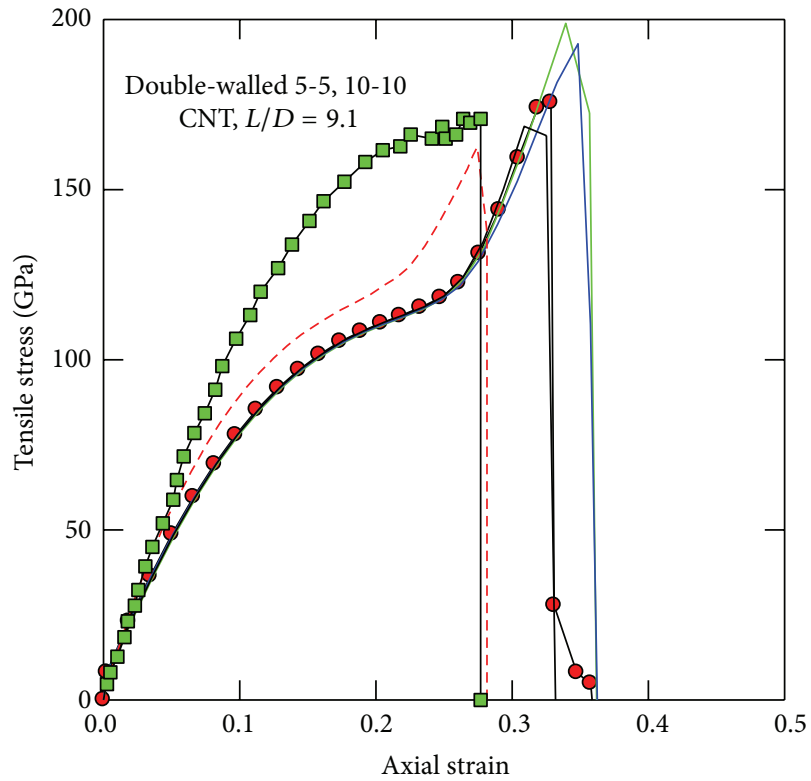

- Airebo potential [12] _ Inclusion $L J$ with Rebo potential [11] Rebo potential [11] _- - Tersoff potential [10]

$\longrightarrow$ Rebo potential [15] $\rightarrow-$ Liew et al. [14]

(a)

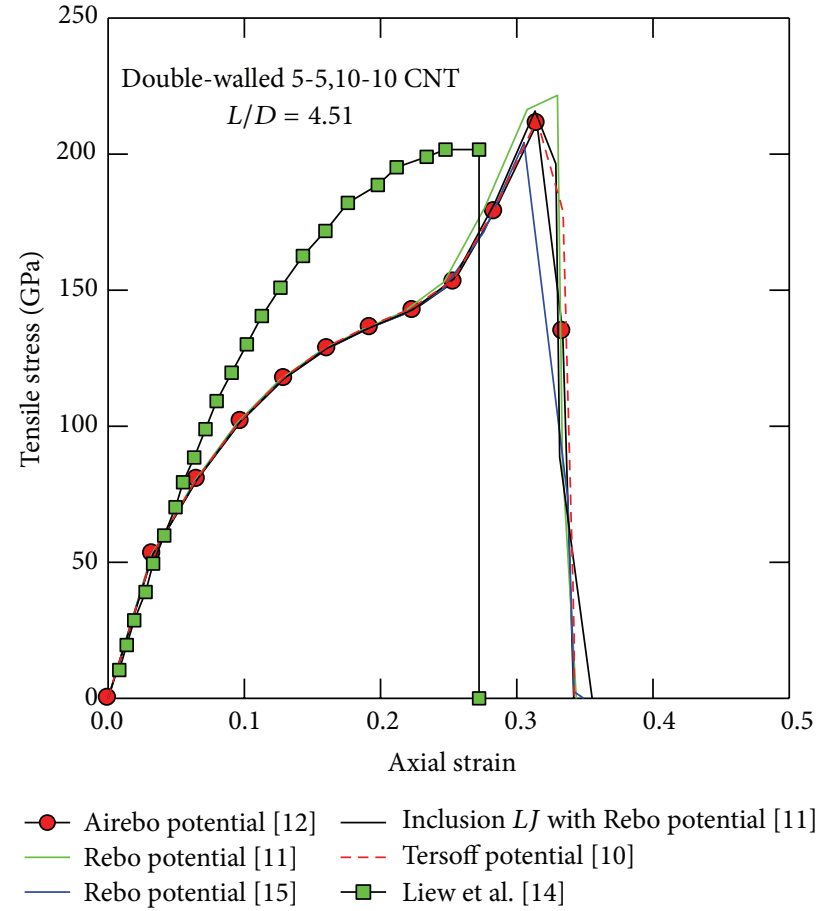

(b)

FIGURE 8: Comparison of the elastic, elastic-plastic response of a double-walled 5-5, 10-10 CNT using different potentials with the published results: (a) $L / D=9.1$; (b) $L / D=4.51$.

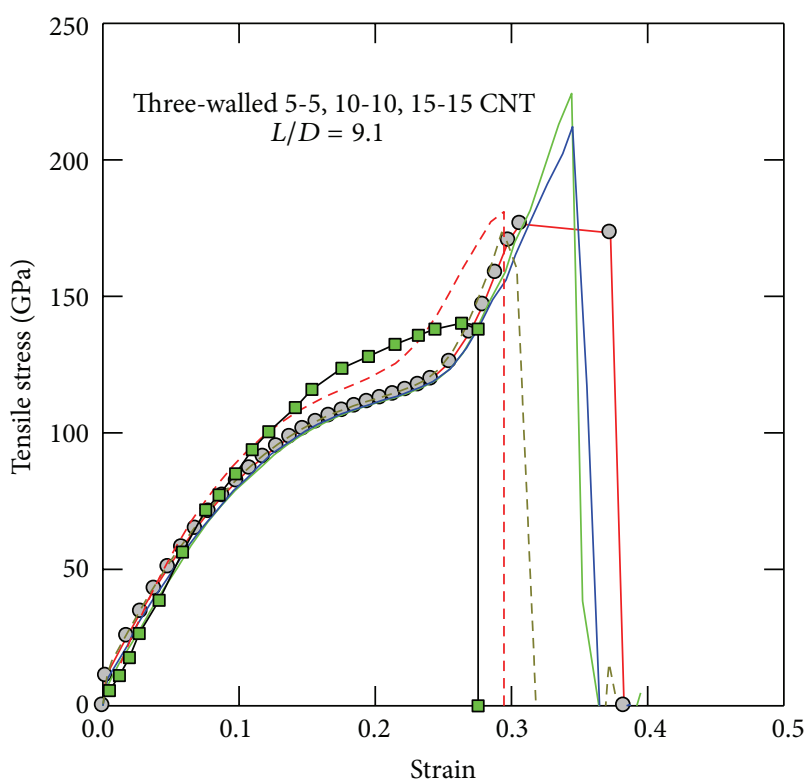

- Airebo potential [12] - - - Inclusion $L J$ with Rebo potential [11]

_ Rebo potential [11] - - - Tersoff potential [10]

$\longrightarrow$ Rebo potential [15] $\rightarrow-$ Liew et al. [14]

(a)

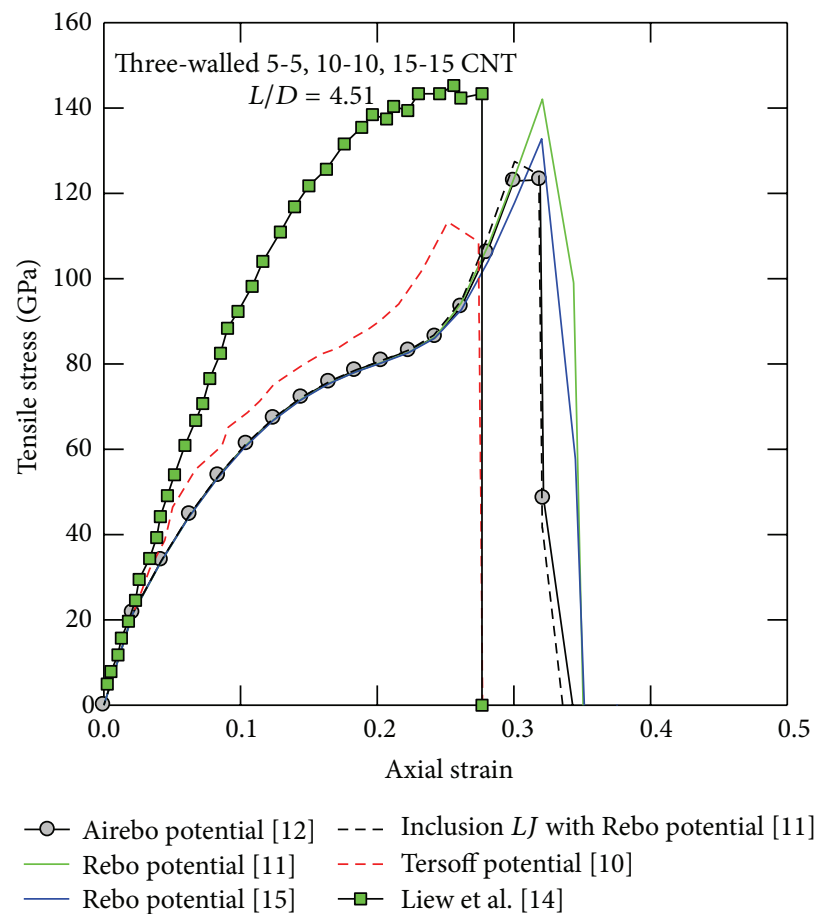

(b)

Figure 9: Comparison of the elastic, elastic-plastic response of a three-walled 5-5, 10-10, and 15-15 CNT using different potentials with the published results: (a) $L / D=9.1$; (b) $L / D=4.51$. 
TABLE 2: Elastic, elastic-plastic properties of a single-walled CNT by the tension test (tension test is carried out by applying velocity of some atoms at both ends of the CNT, $20 \mathrm{~m} / \mathrm{s}$ ).

\begin{tabular}{|c|c|c|c|c|c|c|c|c|}
\hline & $L / D$ & Potentials & $E, \mathrm{GPa}$ & $\sigma_{y}, \mathrm{GPa}$ & $\varepsilon_{y}$ & $\sigma_{u}, \mathrm{GPa}$ & $\varepsilon_{u}$ & $v$ \\
\hline \multirow{10}{*}{ Single-walled 12-12 CNT } & \multirow{5}{*}{9.1} & Airebo [29] & 912.20 & 112.8916 & 0.223 & 169.7363 & 0.3167 & 0.35680 \\
\hline & & Tersoff [10] & 1097.5 & 115.0750 & 0.1754 & 155.32130 & 0.26110 & 0.26110 \\
\hline & & Rebo [11] & 883.42 & 110.8912 & 0.2136 & 214.5082 & 0.357 & 0.35457 \\
\hline & & Rebo [31] & 896.68 & 112.6569 & 0.225 & 196.18111 & 0.35500 & - \\
\hline & & Inclusion $L J$ with Rebo [11] & 897.92 & 114.5128 & 0.2342 & 181.86681 & 0.31920 & - \\
\hline & \multirow{5}{*}{4.51} & Airebo [29] & 935.07 & 114.45180 & 0.23530 & 161.25031 & 0.31590 & 0.29046 \\
\hline & & Tersoff & 1107.7 & 120.3995 & 0.2005 & 147.7876 & 0.2697 & 0.1744 \\
\hline & & Rebo [11] & 899.77 & 111.47040 & 0.21830 & 196.81650 & 0.34170 & 0.26955 \\
\hline & & Rebo [31] & 920.77 & 113.98380 & 0.23500 & 194.24210 & 0.35590 & \\
\hline & & Inclusion $L J$ with Rebo [11] & 913.15 & 114.63750 & 0.23670 & 172.87730 & 0.31780 & \\
\hline \multirow{10}{*}{ Single-walled 10-10 CNT } & \multirow{5}{*}{9.1} & Airebo [29] & 1038.4 & 113.29630 & 0.21190 & 172.72411 & 0.29830 & 0.22345 \\
\hline & & Tersoff [10] & 1236.9 & 120.2628 & 0.1903 & 173.3022 & 0.2713 & 0.1535 \\
\hline & & Rebo [11] & 1036.5 & 110.81970 & 0.19820 & 232.28951 & 0.34530 & 0.3350 \\
\hline & & Rebo [31] & 1040.1 & 113.08620 & 0.21250 & 212.39920 & 0.34550 & \\
\hline & & Inclusion $L J$ with Rebo [11] & 1040.5 & 113.66160 & 0.21330 & 183.36310 & 0.29990 & \\
\hline & \multirow{5}{*}{4.51} & Airebo & 1083.9 & 116.69020 & 0.22680 & 199.44099 & 0.32050 & 0.18104 \\
\hline & & Tersoff & 1120.4 & 118.0722 & 0.1862 & 191.8131 & 0.3041 & 0.2267 \\
\hline & & Rebo [11] & 1084.4 & 111.19750 & 0.19570 & 247.91631 & 0.35530 & \\
\hline & & Rebo [31] & 1084.1 & 116.29670 & 0.22620 & 222.50110 & 0.35370 & \\
\hline & & Inclusion $L J$ with Rebo [11] & 1088.9 & 111.67250 & 0.19620 & 211.41220 & 0.32130 & 0.3637 \\
\hline
\end{tabular}

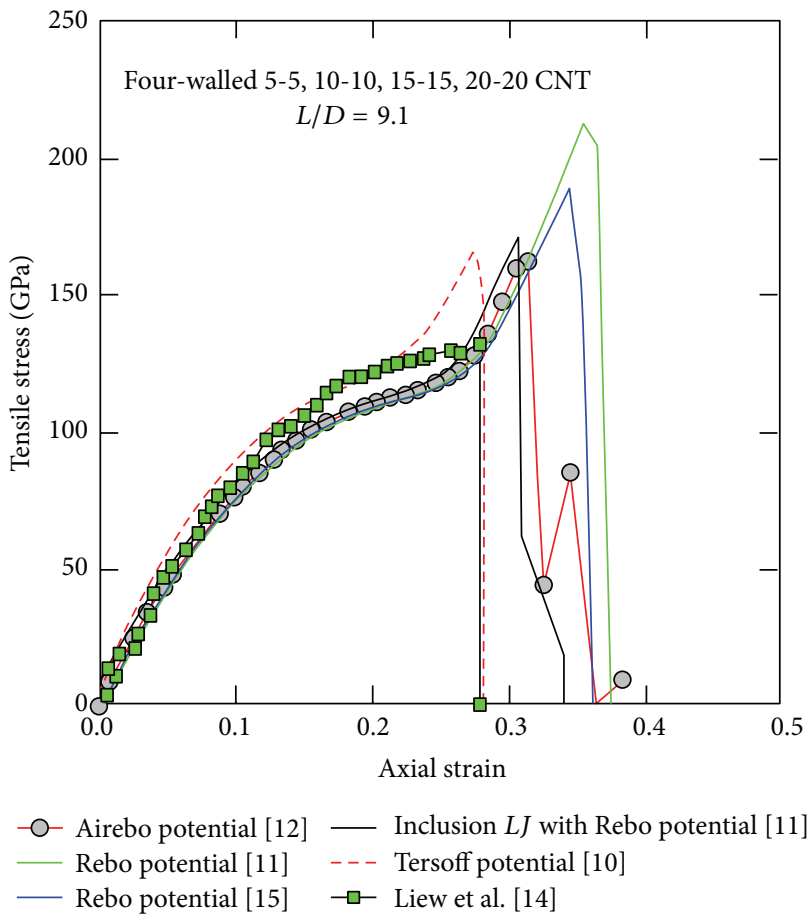

(a)

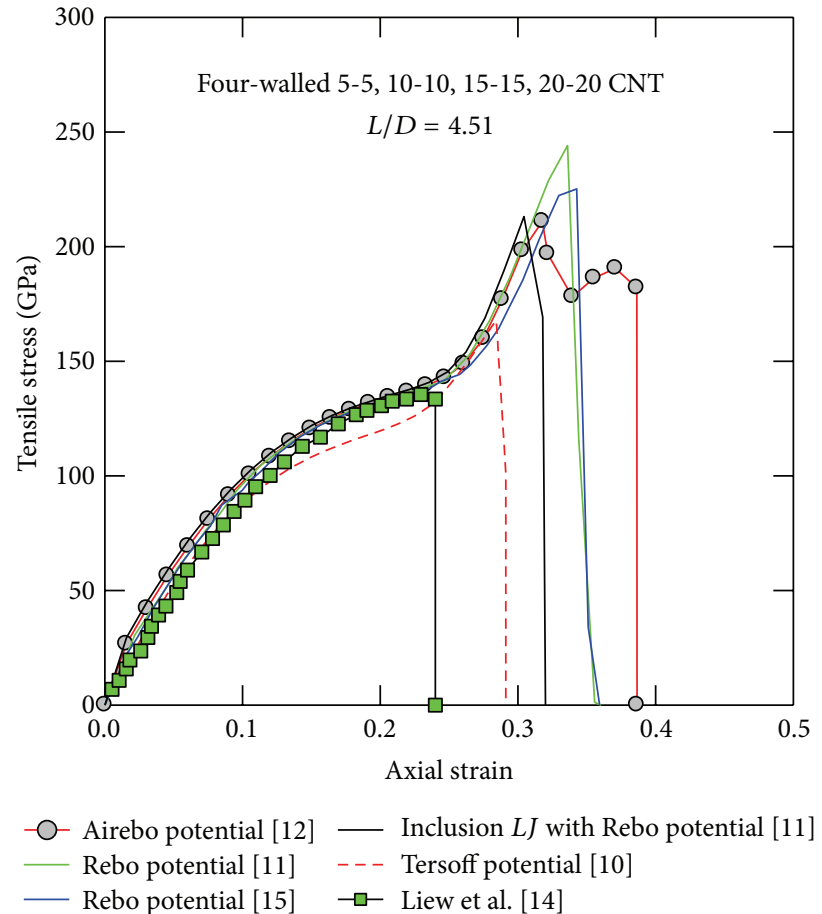

(b)

FIGURE 10: Comparison of the elastic, elastic-plastic response of a four-walled, 5-5, 10-10, 15-15, and 20-20 CNT using different potentials with the published results: (a) $L / D=9.1$; (b) $L / D=4.51$. 
TABLE 3: Elastic, elastic-plastic properties of multiwall CNT by the tension test (tension test is carried out by applying velocity of some atoms at both ends of the CNT, $20 \mathrm{~m} / \mathrm{s}$ ).

\begin{tabular}{|c|c|c|c|c|c|c|c|}
\hline & $L / D$ & Potentials & $E, \mathrm{GPa}$ & $\sigma_{y}, \mathrm{GPa}$ & $\varepsilon_{y}$ & $\sigma_{u}, \mathrm{GPa}$ & $\varepsilon_{u}$ \\
\hline \multirow{10}{*}{ Double-walled 5-5, 10-10 CNT } & \multirow{5}{*}{9.1} & Airebo [29] & 1014.5 & 112.82160 & 0.21750 & 175.50349 & 0.32870 \\
\hline & & Tersoff [10] & 1152.9 & 112.3452 & 0.16460 & 162.50680 & 0.27450 \\
\hline & & Rebo [11] & 970.41 & 112.63770 & 0.22090 & 198.80260 & 0.33950 \\
\hline & & Rebo [31] & 1000.9 & 112.44860 & 0.21870 & 192.85950 & 0.34840 \\
\hline & & Inclusion $L J$ with Rebo [11] & 983.05 & 113.17720 & 0.22080 & 168.55659 & 0.30890 \\
\hline & \multirow{5}{*}{4.51} & Airebo [29] & 1047 & 113.95940 & 0.22380 & 168.98421 & 0.31490 \\
\hline & & Tersoff $[10]$ & 1053.6 & 114.49510 & 0.22250 & 170.08620 & 0.31500 \\
\hline & & Rebo [11] & 1063.8 & 113.55300 & 0.21690 & 177.22290 & 0.32990 \\
\hline & & Rebo [31] & 1061.7 & 113.04890 & 0.21790 & 163.33620 & 0.30550 \\
\hline & & Inclusion $L J$ with Rebo [11] & 1045.1 & 114.15500 & 0.22350 & 172.63960 & 0.31340 \\
\hline \multirow{10}{*}{ Three-walled 5-5, 10-10, 15-15 CNT } & \multirow{5}{*}{9.1} & Airebo [29] & 1071.2 & 114.03640 & 0.21340 & 176.42340 & 0.30660 \\
\hline & & Tersoff & 1112.1 & 117.7347 & 0.1846 & 180.9215 & 0.2946 \\
\hline & & Rebo [11] & 960.32 & 113.11430 & 0.21650 & 224.30141 & 0.34430 \\
\hline & & Rebo [31] & 1011.3 & 111.75010 & 0.20460 & 212.10780 & 0.34500 \\
\hline & & Inclusion $L J$ with Rebo [11] & 1118.5 & 110.95180 & 0.19090 & 174.31090 & 0.29360 \\
\hline & \multirow{5}{*}{4.51} & Airebo [29] & 757.97 & 80.74040 & 0.20330 & 123.15620 & 0.31920 \\
\hline & & Tersoff [10] & 917.49 & 84.03300 & 0.17470 & 108.58530 & 0.27420 \\
\hline & & Rebo [11] & 755.7 & 82.83280 & 0.22410 & 142.03690 & 0.32090 \\
\hline & & Rebo [31] & 756.59 & 80.30010 & 0.20370 & 117.80770 & 0.30060 \\
\hline & & Include $L J$ with Rebo [11] & 759.4 & 80.85670 & 0.20450 & 127.86900 & 0.30110 \\
\hline \multirow{10}{*}{ Four-walled 5-5, 10-0, 15-15, 20-20 CNT } & \multirow{5}{*}{9.1} & Airebo [29] & 956.18 & 113.64290 & 0.22340 & 162.12520 & 0.31360 \\
\hline & & Tersoff [10] & 1162.3 & 117.3119 & 0.1849 & 166.3823 & 0.2750 \\
\hline & & Rebo [11] & 832.63 & 111.00820 & 0.21450 & 213.49680 & 0.35470 \\
\hline & & Rebo [31] & 877.48 & 111.14260 & 0.21370 & 189.54539 & 0.34470 \\
\hline & & Inclusion $L J$ with Rebo [11] & 1053 & 113.42000 & 0.21490 & 171.45770 & 0.31030 \\
\hline & \multirow{5}{*}{4.51} & Airebo [29] & 1222 & 136.58340 & 0.21970 & 190.49490 & 0.37090 \\
\hline & & Tersoff [10] & 1052.8 & 116.5393 & 0.1853 & 167.8656 & 0.2843 \\
\hline & & Rebo [11] & 1174.4 & 133.39349 & 0.20440 & 244.05009 & 0.33610 \\
\hline & & Rebo [31] & 1150 & 136.24100 & 0.22530 & 225.16690 & 0.34280 \\
\hline & & Inclusion $L J$ with Rebo [11] & 1273.8 & 137.69620 & 0.22140 & 213.03430 & 0.30450 \\
\hline
\end{tabular}

till fracture. In the outer layer of a four-walled CNT, how 57-7-5 defects generate during fracture which are reflected in Figure 11(c).

Table 2 lists the computed Young's modulus, poison's ration, yield strength, ultimate strength, yield strain, and ultimate strain of single-walled 12-12 and 10-10 CNTs with length-to-diameter ratios equal to 9.1 and 4.51, respectively, using five different potentials. Young's modulus, yield strength, and yield strain of single-walled 12-12 CNTs having aspect ratio 9.1 obtained by five different potentials separately are lower than those of aspect ratio 4.51, but the opposite phenomenon is observed for its ultimate strength because per atom potential energy change with strain is higher for CNTs with lower aspect ratio than from its higher aspect ratio. Young's modulus and yield strength of a single-walled 10-10 CNT having aspect ratio 9.1 obtained by Rebo potential [11], Rebo potential [31], and Airebo potential [29] are lower than those of aspect ratio 4.51; on the other hand, ultimate strength and strain having aspect ratio 9.1 obtained by Rebo potential [11], Rebo potential [31], Airebo potential [29],
Inclusion $L J$ with Rebo potential [11], and Tersoff potential [10] are lower than those of aspect ratio 4.51, separately. Per atom potential energy with strain is dramatically changed due to change of aspect ratio of CNTs which change the slope of stress strain curve of CNTs subjected to a tensile load using different potentials and for which effects of aspect ratio are observed on the elastic, elastic-plastic properties of CNTs. From Table 2, it is observed that Young's modulus, yield strength, yield strain, ultimate strength, and ultimate strain of 12-12 single-walled CNTs with the same aspect ratio differ from those of single-walled 10-10 CNTs due to the size effect in diameters because diameter of single-walled 12-12 CNT is higher than that of single-walled 10-10 CNTs. Effects of different potentials on Young's modulus, yield strength, yield strain, ultimate strength, ultimate strain, and poison's ration of single-walled 12-12 and 10-10 CNTs are also observed in the tabular results.

Table 3 lists the computed Young's modulus, yield strength, ultimate strength, yield strain, and ultimate strain of double-walled, three-walled, and four-walled CNTs with 


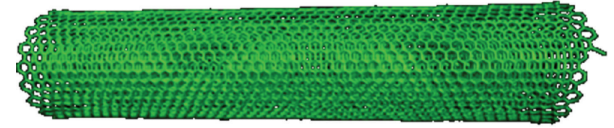

(a)

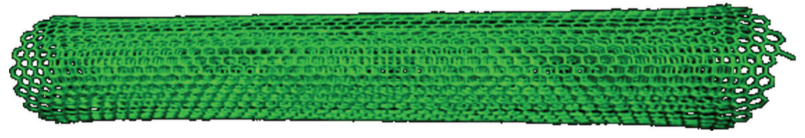

(b)

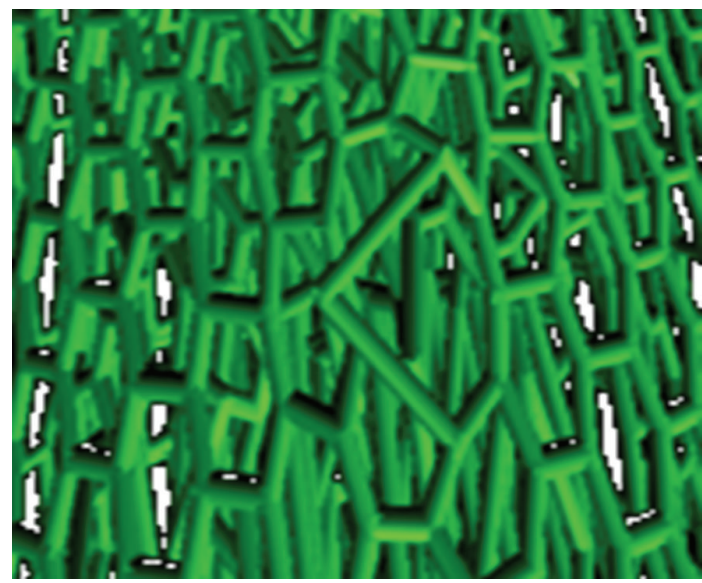

(c)

FIGURE 11: Physical configuration of CNT at different state: (a) original configuration; (b) before fracture; (c) 5-7-7-5 defects during fracture of a four-walled, 5-5, 10-10, 15-15, and 20-20 CNT $(L / D=$ 4.51).

length-to-diameter ratios 9.1 and 4.51, respectively, using five different potentials with the published results. Young's moduli of double-walled CNT having aspect ratio 9.1 obtained by Airebo potential [29], Rebo potential [11], Rebo potential [31], and Inclusion $L J$ with Rebo potential [11] are lower than those of aspect ratio 4.51, separately, but yield strengths of a double-walled CNT having aspect ratio 4.51 using Rebo potential [11], Rebo potential [31], Airebo potential [29], Inclusion $L J$ with Rebo potential [11], and Tersoff potential [10] are higher than those of aspect ratio 9.51, separately. Yield strains, ultimate strengths, and ultimate strains of doublewalled CNTs having aspect ratio 4.51 using Rebo potential [11], Rebo potential [31], and Airebo potential [29] are lower than those of aspect ratio 9.1. Although Young's modulus of CNTs with aspect ratio 9.1 obtained by Tersoff potential is higher than that of aspect ratio 4.51, for yield strength, yield strain, ultimate strength, and ultimate strain, the opposite phenomenon is observed. Young's moduli, yield strengths, and ultimate strengths of three-walled CNT with aspect ratio 9.1 obtained by all potentials mentioned in the Table 3 are higher than those of aspect ratio 4.51, separately. Yield strain and ultimate strain of three-walled CNTs obtained by Rebo potential [11], Rebo potential [31], Inclusion $L J$ with Rebo potential [11], and Airebo potential [29] mentioned in Table 3 also show the effect of aspect ratio of CNTs. Young's moduli, yield strengths, ultimate strengths of four-walled CNT with aspect ratio 4.51 obtained by all potentials mentioned in the Table 3 are higher than those of aspect ratio 9.1, separately. There are also observed effects of aspect ratio on the yield strains and yield strengths of four-walled CNTs obtained by all potentials mentioned in Table 3. From Table 3, it is clearly observed that Young's moduli, yield strengths, yield strains, ultimate strengths, and ultimate strains of doublewalled, three-walled, and four-walled CNTs with aspect ratios 9.1 and 4.51 obtained by Airebo potential [29] and Inclusion $L J$ with Rebo potential [11] differ from those of all of the other potentials due to layering effects of CNTs because in Airebo potential [29] and Inclusion $L J$ with Rebo potential [11], Van der Waals interaction is added which interact with interlayer carbon atoms during loading. Besides, From Tables 2 and 3, it is clearly observed that Young's moduli, yield strengths, yield strains, ultimate strengths, and ultimate strains of singlewalled, double-walled, three-walled, and four-walled CNTs with aspect ratios 9.1 and 4.51 obtained by Rebo potential [31] and Airebo potential [29] differ from those of all of the other potentials due to torsional effects of $\mathrm{C}-\mathrm{C}$ bonds of CNTs because, in Rebo potential [31] and Airebo potential [29], torsional interaction is added which include angular movement of $\mathrm{C}-\mathrm{C}$ bond during loading.

From the tabular results, it is clearly observed that Young's modulus of single-walled, double-walled, three-walled, and four-walled CNTs with $L / D$ ratios 9.1 and 4.51, respectively, obtained by Tersoff potential [10] is greater than that obtained by Rebo potential [11], Rebo potential [31], Inclusion LJ with Rebo potential [11], and Airebo potential [29] because the slope of per atom potential energy with strain of CNTs obtained by Tersoff potential is higher than those of other potentials (see Figures 2 and 5). On the other hand, the ultimate strength of single-walled, double-walled, threewalled, and four-walled CNTs with $L / D$ ratios 9.1 and 4.51, respectively, obtained by Rebo potential [11] is greater than that obtained by Tersoff potential [10], Rebo potential [31], Inclusion $L J$ with Rebo potential [11], and Airebo potential [29] because per atom potential energy of CNTs with strain obtained by Rebo potential is higher than those of other potentials and it takes the highest stress and strain to reach minimum per atom potential energy for the breaking of CNTs during tensile loading using Rebo potential [11] (see Figures 2 and 5). For single-, double-, three-, and four-walled CNTs, the ultimate strength obtained by Airebo [29] potential is lower than that of Rebo potential [11] because in Airebo potential [29] torsional and Van der Waals interactions are present which reduce per atom potential energy for which ultimate strength of CNTs obtained by Airebo potential [29] is lower as compared to Rebo potential [11]. For single-, double-, three-, and four-walled CNTs, the effects of torsion and Van der Waals interactions are observed clearly when their ultimate strength obtained by Rebo potential [11] is compared with that of Rebo potential [31], Inclusion $L J$ with Rebo potential [11], and Airebo potential [29]. From the tabular results, it is clear that torsion and Van der Waals interactions play an important role in their mechanical properties in the plastic range during loading.

When tensile load is applied at its both ends of CNTs using different potentials by molecular dynamics simulation, 
per atom potential energy decreases and during fracture per atom potential energy reaches a minimum value. As per atom potential energy of $\mathrm{C}-\mathrm{C}$ structure using Tersoff potential is lower (Table 1 in [33]) than that of Rebo potential [11] and Rebo potential [31], CNT will break at lower strain than that of other potentials and this phenomenon is already reflected in the above graphical and Tabular results. As bond radius and bond length of $\mathrm{C}-\mathrm{C}$ in Tersoff potential are higher (see Table 1 in [33]) than those of Rebo [11] and Rebo [31] potential, the stiffness of CNT and the slope of the stress strain curves using Tersoff potential should be higher than those of Rebo potential [11] and Rebo potential [31] and this phenomenon is clearly observed in the graphical and Tabular results. As per atom potential energy of C-C in Rebo potential [11] is higher (see Table 1 in [33]) than that of Rebo [31] and Tersoff potential [10], the ultimate strength of CNT using Rebo potential [11] should be higher than that of Rebo [31] and Tersoff potential [10] because to reach minimum per atom potential energy for its breaking, it needs the highest tensile stress among all of the other potentials and from the graphical results, this phenomenon is also reflected in the graphical and tabular results. When Van der Waals interaction is present in Airebo potential [29], during tension some neighboring carbon atoms come in repulsive and attractive zone. As a result, $\mathrm{C}$ atoms experience extra stress during loading which enhance decreasing the ultimate strength of CNT. The other reason is that per atom potential energy of CNT structure obtained by Airebo potential [29] is lower than that of Rebo potential [11] and greater than that of Tersoff potential [10]. So for this reason, the ultimate strength of different types CNT using Airebo potential [29] is higher than that of Tersoff potential [10] and less than that of Rebo potential [11] which is reflected in the graphical and Tabular results.

From the tabular results as shown in Tables 2 and 3, the effects of layering, length-to-diameter ratio of CNTs on their elastic, elastic-plastic properties are clearly observed when the tension tests are carried out using different potentials. Besides, there is an observed effect of empirical potentials on Young's modulus, yield strength, yield strain, ultimate strength, ultimate strain, and Poisson's ratio of CNT under tension test using molecular dynamics simulation. Effects of potentials on the reliability and correctness of the solution are also reflected in the graphical and tabular results. Effects of layers and length-to-diameter ratio of CNT are also reflected in the tabular results.

\section{Conclusions}

We study per atom potential energy of single-walled CNT subjected to axial tensile load at its opposite ends at a very low strain rate using Rebo potential [11], Rebo potential [31], Airebo potential [29], and Tersoff potential [10]. We compare the stress strain relationship of single-walled 12-12 CNTs obtained by Rebo potential [11], Rebo potential [31], Airebo potential [29], and Tersoff potential [10] at a very low strain rate with that of Belytschko et al. [30] obtained by density functional theory and the stress strain relationship obtained by Rebo potential [11] and Airebo potential [29] agree with that of Belytschko et al. [30]. At high strain rate we also study the elastic, elastic-plastic properties of single and multiwalled CNT subjected to uniform tension at its opposite ends using five different potentials such as Rebo potential [11], Rebo potential [31], Inclusion $L J$ with Rebo potential [11], Tersoff potential [10], and Airebo potential [29] with the published results as a comparative manner to understand the effect of potentials on the solution. From the comparative study it is clearly understood that the reliability and accuracy of Airebo potential [29] are higher than other potentials for the elastic, elastic-plastic analysis of CNT because in Airebo potential [29] long range Van der Waals interaction and torsional effect of bond are included. To test the reliability of elastic, elasticplastic properties of CNTs obtained by us using different potentials, we compared our results with some published results and found out major limitations of such results which will be helpful for the further study of the elastic, elasticplastic properties of CNTs under tension. Effects of number of layers, length-to-diameter ratio, and empirical potentials are also observed on the elastic, elastic-plastic properties of CNTs.

\section{Conflict of Interests}

The authors declare that they have no conflict of interests regarding the publication of this paper.

\section{References}

[1] S. Lijima and T. Lchihashi, "Single-shell carbon nanotubes of 1-nm diameter," Nature, vol. 363, no. 6430, pp. 603-605, 1993.

[2] D. S. Bethune, C. H. Kiang, M. S. De Vries et al., "Cobaltcatalysed growth of carbon nanotubes with single-atomic-layer walls," Nature, vol. 363, no. 6430, pp. 605-607, 1993.

[3] S. Iijima, "Helical microtubules of graphitic carbon," Nature, vol. 354, no. 6348, pp. 56-58, 1991.

[4] T. W. Ebbesen and P. M. Ajayan, "Large-scale synthesis of carbon nanotubes," Nature, vol. 358, no. 6383, pp. 220-222, 1992.

[5] L. Merhari, Hybrid Nanocomposites for Nanotechnology: Electronic, Optical, Magnetic and Biomedical Applications, Springer, New York, NY, USA, 2009.

[6] O. Breuer and U. Sundararaj, "Big returns from small fibers: a review of polymer/carbon nanotube composites," Polymer Composites, vol. 25, no. 6, pp. 630-645, 2004.

[7] B. I. Yakobson, C. J. Brabec, and J. Bernholc, "Nanomechanics of carbon tubes: instabilities beyond linear response," Physical Review Letters, vol. 76, pp. 2511-2524, 1996.

[8] A. Garg, J. Han, and S. B. Sinnott, "Interactions of carbonnanotubule proximal probe tips with diamond and graphene," Physical Review Letters, vol. 81, no. 11, pp. 2260-2263, 1998.

[9] B. I. Yakobson, M. P. Campbell, C. J. Brabec, and J. Bernholc, "High strain rate fracture and C-chain unraveling in carbon nanotubes," Computational Materials Science, vol. 8, no. 4, pp. 341-348, 1997.

[10] J. Tersoff, "New empirical approach for the structure and energy of covalent systems," Physical Review B, vol. 37, no. 12, pp. 6991$7000,1988$.

[11] D. W. Brenner, "Empirical potential for hydrocarbons for use in simulating the chemical vapor deposition of diamond films," Physical Review B, vol. 42, no. 15, pp. 9458-9471, 1990. 
[12] S. B. Sinnott, O. A. Shenderova, C. T. White, and D. W. Brenner, "Mechanical properties of nanotubule fibers and composites determined from theoretical calculations and simulations," Carbon, vol. 36, no. 1-2, pp. 1-9, 1998.

[13] K. M. Liew, X. Q. He, and C. H. Wong, "On the study of elastic and plastic properties of multi-walled carbon nanotubes under axial tension using molecular dynamics simulation," Acta Materialia, vol. 52, no. 9, pp. 2521-2527, 2004.

[14] P. M. Agrawal, B. S. Sudalayandi, L. M. Raff, and R. Komanduri, "Molecular dynamics (MD) simulations of the dependence of $\mathrm{C}-\mathrm{C}$ bond lengths and bond angles on the tensile strain in single-wall carbon nanotubes (SWCNT)," Computational Materials Science, vol. 41, no. 4, pp. 450-456, 2008.

[15] C. H. Wong and V. Vijayaraghavan, "Nanomechanics of imperfectly straight single walled carbon nanotubes under axial compression by using molecular dynamics simulation," Computational Materials Science, vol. 53, no. 1, pp. 268-277, 2012.

[16] K. I. Tserpes and P. Papanikos, "Finite element modeling of single-walled carbon nanotubes," Composites Part B, vol. 36, no. 5, pp. 468-477, 2005.

[17] J.-P. Salvetat-Delmotte and A. Rubio, "Mechanical properties of carbon nanotubes: a fiber digest for beginners," Carbon, vol. 40, no. 10, pp. 1729-1734, 2002.

[18] B. Coto, I. Antia, M. Blanco et al., "Molecular dynamics study of the influence of functionalization on the elastic properties of single and multiwall carbon nanotubes," Computational Materials Science, vol. 50, no. 12, pp. 3417-3424, 2011.

[19] A. Mashreghi and M. M. Moshksar, "Bond lengths and bond angles of armchair single-walled carbon nanotubes through molecular dynamics and potential energy curve approaches," Computational Materials Science, vol. 49, no. 4, pp. 871-875, 2010.

[20] W. Tang, M. H. Santare, and S. G. Advani, "Melt processing and mechanical property characterization of multi-walled carbon nanotube/high density polyethylene (MWNT/HDPE) composite films," Carbon, vol. 41, no. 14, pp. 2779-2785, 2003.

[21] M. Sammalkorpi, A. Krasheninnikov, A. Kuronen, K. Nordlund, and K. Kaski, "Mechanical properties of carbon nanotubes with vacancies and related defects," Physical Review B, vol. 70, no. 24, Article ID 245416, 8 pages, 2005.

[22] Q. Wang, J. Dai, W. Li, Z. Wei, and J. Jiang, "The effects of CNT alignment on electrical conductivity and mechanical properties of SWNT/epoxy nanocomposites," Composites Science and Technology, vol. 68, no. 7-8, pp. 1644-1648, 2008.

[23] A. Allaoui, S. Bai, H. M. Cheng, and J. B. Bai, "Mechanical and electrical properties of a MWNT/epoxy composite," Composites Science and Technology, vol. 62, no. 15, pp. 1993-1998, 2002.

[24] J.-P. Salvetat, J.-M. Bonard, N. B. Thomson et al., "Mechanical properties of carbon nanotubes," Applied Physics A, vol. 69, no. 3, pp. 255-260, 1999.

[25] Y. J. Liu and X. L. Chen, "Evaluations of the effective material properties of carbon nanotube-based composites using a nanoscale representative volume element," Mechanics of Materials, vol. 35, no. 1-2, pp. 69-81, 2003.

[26] A. Hernández-Pérez and F. Avilés, "Modeling the influence of interphase on the elastic properties of carbon nanotube composites," Computational Materials Science, vol. 47, no. 4, pp. 926-933, 2010.

[27] S. K. Deb Nath, H. Tohmyoh, and M. A. Salam Akanda, "Evaluation of elastic, elastic-plastic properties of thin Pt wire by mechanical bending test," Applied Physics A, vol. 103, no. 2, pp. 493-496, 2011.
[28] S. K. Deb Nath and S. -G. Kim, "On the elastic, elastic-plastic properties of Au nanowires in the range of diameters 1-200nm," Journal of Applied Physics, vol. 112, no. 12, Article ID 123522, 10 pages, 2012.

[29] S. J. Stuart, A. B. Tutein, and J. A. Harrison, "A reactive potential for hydrocarbons with intermolecular interactions," Journal of Chemical Physics, vol. 112, no. 14, pp. 6472-6486, 2000.

[30] T. Belytschko, S. P. Xiao, G. C. Schatz, and R. S. Ruoff, "Atomistic simulations of nanotube fracture," Physical Review B, vol. 65, no. 23, Article ID 235430, 8 pages, 2002.

[31] D. W. Brenner, O. A. Shenderova, J. A. Harrison, S. J. Stuart, B. $\mathrm{Ni}$, and S. B. Sinnott, "A second-generation reactive empirical bond order (REBO) potential energy expression for hydrocarbons," Journal of Physics: Condensed Matter, vol. 14, no. 4, pp. 783-802, 2002.

[32] W. H. Pres, B. P. Flannery, S. A. Teukoisky, and W. T. Vattering, Numerical Recipes, Cambridge University Press, Cambridge, UK, 1986.

[33] R. Smith and K. Beardmore, "Molecular dynamics studies of particle impacts with carbon-based materials," Thin Solid Films, vol. 272, no. 2, pp. 255-270, 1996. 

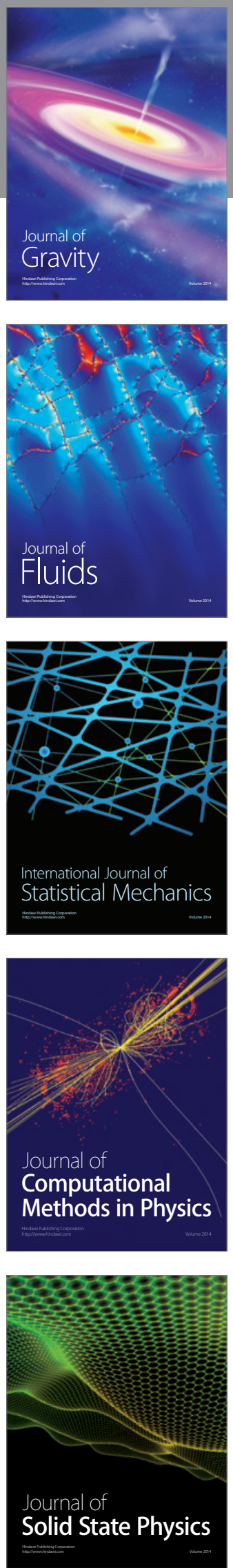

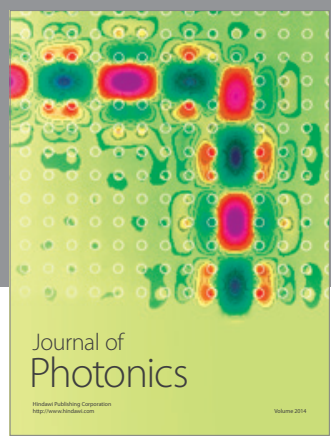

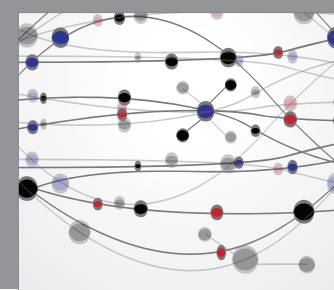

The Scientific World Journal

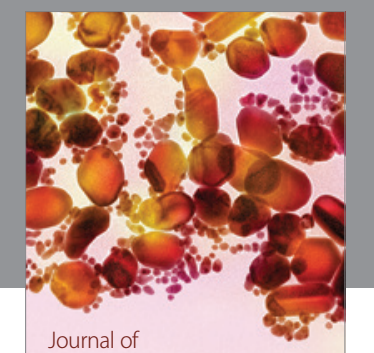

Soft Matter
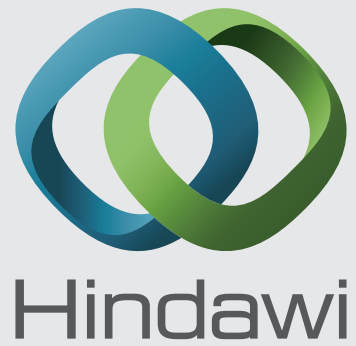

Submit your manuscripts at

http://www.hindawi.com
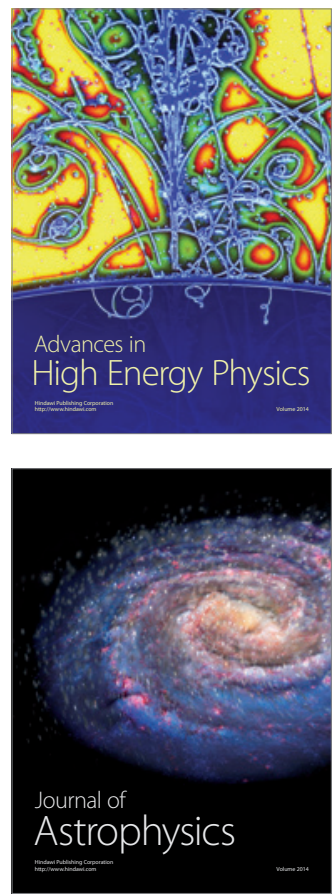
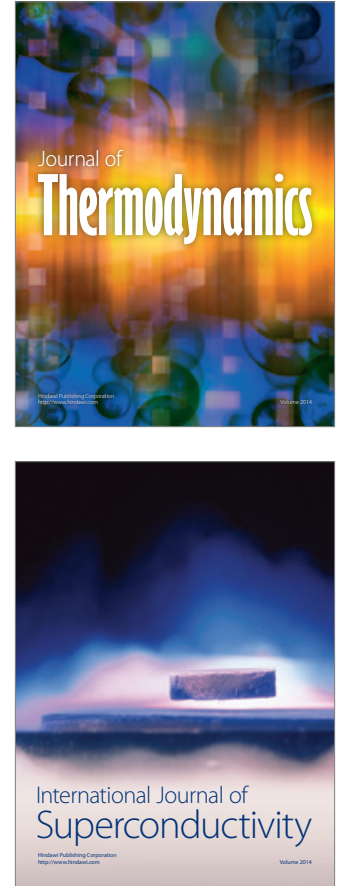
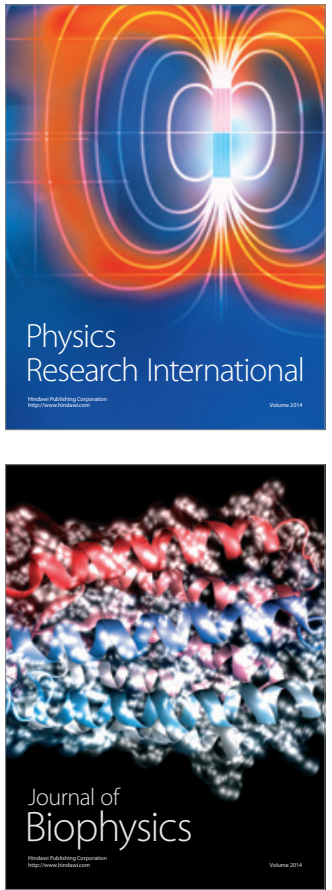
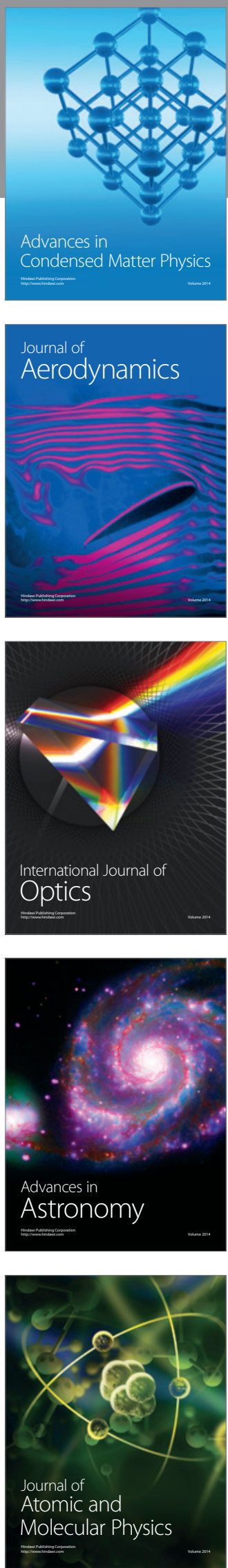